\title{
Normalization of TanDEM-X DSM Data in Urban Environments With Morphological Filters
}

\author{
Christian Geiß, Michael Wurm, Markus Breunig, Andreas Felbier, and Hannes Taubenböck
}

\begin{abstract}
The TanDEM-X mission (TDM) is a spaceborne radar interferometer which delivers a global digital surface model (DSM) with an unprecedented spatial resolution. This allows resolving objects above ground such as buildings. Extracting and characterizing those objects in an automated manner represents a challenging problem but opens simultaneously a broad range of large-area applications. In this paper, we discuss and evaluate the suitability of morphological filters (MFs) for the derivation of normalized DSMs from the TDM in complex urban environments and introduce a novel region-growing-based progressive MF procedure. This approach is jointly proposed and can be combined with a postclassification processing scheme to specifically allow for a viable reconstruction of urban morphology in a challenging terrain. The filter approach comprises a multistep procedure using concepts of morphological image filtering, region growing, and interpolation techniques. Therefore, it extends the idea of progressive MFs. The latter aim to identify nonground pixels in the DSM by gradually increasing the size of a structuring element and applying iteratively an elevation difference threshold. After the identification of initial nonground pixels, here, potential nonground pixels are identified within each iteration, and their similarity with respect to neighboring nonground pixels is assessed. Pixels are finally labeled as nonground if a constraint is fulfilled. The postclassification processing scheme adapts techniques of object-based image analyses to further refine regions of classified nonground pixels. Digital terrain models are subsequently generated by interpolating between identified ground pixels. Experimental results are obtained for settlement areas that cover large parts of the cities of Izmir (Turkey) and Wuppertal (Germany). They confirm the capability of the proposed approaches for a reduction of omission errors compared to basic MF-based methods when classifying ground pixels, which is favorable in a mountainous terrain with steep slopes.
\end{abstract}

Index Terms-Digital surface models (DSM), digital terain models (DTM), morphological filters, TanDEM-X.

\section{INTRODUCTION}

$\mathbf{T}$ HE TanDEM-X mission (TDM) acquires data for a global digital surface model (DSM) with unprecedented resolu-

Manuscript received May 7, 2014; revised October 22, 2014 and January 9, 2015; accepted January 21, 2015. This work was supported in part by Helmholtz-EOS (Earth Observation System) and by the European Commission's Seventh Framework Programme [FP7/2007-2013] under Grant agreement no. 312972 "Framework to integrate Space-based and in situ sENSing for dynamic vUlnerability and recover Monitoring."

The authors are with the German Aerospace Center (DLR), German Remote Sensing Data Center (DFD), 82234 Weßling, Germany (e-mail: christian. geiss@dlr.de; michael.wurm@dlr.de; markus.breunig@dlr.de; andreas. felbier@dlr.de; hannes.taubenboeck@dlr.de).

Color versions of one or more of the figures in this paper are available online at http://ieeexplore.ieee.org.

Digital Object Identifier 10.1109/TGRS.2015.2396195 tion characteristics [1]. The pixel spacing of 0.4 arcseconds $(\sim 12 \mathrm{~m})$ allows resolving objects in urban environments above ground such as buildings. Extraction of those objects opens a broad range of large-area applications, which are, to date, unfeasible due to data availability and costs. In this sense, spatial analyses can be extended by including 3-D characteristics of urban environments and rely on data which are available for large areas consistently. Examples of applications include the analysis of urbanization processes [2]-[4], characterization of urban morphology [5]-[7], and assessment of vulnerability and risk of the built environment [8]-[10], among others.

When aiming at the extraction of those objects in an automated manner, it is common to derive a digital terrain model (DTM) from the DSM first. A DTM contains elevation measurements of the bare Earth (BE) without including objects above ground. A DTM allows computing a normalized DSM (nDSM). The latter then naturally comprises elevation information of objects above ground. Numerous approaches have been postulated to retrieve DTM information from a DSM. Therefore, a large share of approaches is related to the derivation of DTMs from very high resolution DSMs, with ground sampling distances usually smaller than $1 \mathrm{~m}$. These kinds of DSMs are frequently derived from stereoscopic optical acquisitions, interferometric SAR measurements, or laser scanning (LiDAR) [11]-[13]. In particular, the latter has triggered the development of ground filtering algorithms [14]-[18]. However, the lower spatial resolution of the TDM DSM data hampers the use of many approaches.

With the benefit of a very high spatial resolution, for instance, the existence of a distinct difference between the slope of terrain and that of nonground objects such as buildings and trees can be exploited [19], [20]. This prerequisite generally cannot be met by TDM DSM data because of their acquisition characteristics and spatial resolution. Moreover, for this study, we only consider approaches that operate on grayscale images and neglect waveform-related approaches. This is due to useroriented considerations, which include the fact that elevation measurements of the TDM are converted into regular grayscale grid images, including several tailored correction procedures such as masking of water areas, before dissemination. Regarding techniques that are suitable for application on grayscale grid images, approaches based on mathematical morphology [16], [21]-[25] are common due to their simplicity in accordance with good performance. By means of compositions of algebraic set operations [26], objects in the image such as buildings can be identified since their elevation values are usually distinctively higher than the elevation values of surrounding ground measurements. 
However, mathematical morphology-based filters are prone to errors in steep terrains [19], [27], [28]. Generally, the biggest challenge when applying morphological methods is to keep terrain features unchanged while using large sizes of the structuring element (SE) [24]. Ground points are mistakenly treated as nonground points in such settings, which results in an overestimation of objects and their height in the final nDSM [17], [18], [27]. Besides these general challenges, the spatial resolution of the TDM data poses particular challenges to a suitable approach. When aiming at the extraction of urban land cover objects such as buildings, the spatial resolution limits the use of algorithms that are designed for data with ground sampling distances considerably smaller than the objects of interest. In particular, a large share of approaches uses the concept of progressive morphological filters (PMFs) that aim to separate ground/BE from nonground/objects first (e.g., [16], [24], and [25]). Therefore, free parameters can be altered in a progressive manner in dependence of the size of the SE of the MF. This allows coping with different kinds of land cover objects in the data [16], [29]. For instance, low elevation difference thresholds are used for small sizes of the SE to eliminate objects such as bushes, small trees, or cars. However, given the spatial resolution of the TDM data, even the smallest size of the SE $(3 \times 3$ pixels $)$ comprises a size that can already exceed a building. Consequently, the conceptual advancement of a progressive procedure cannot be exploited the same way as it was done in previous studies. Such a setting determines the application of parameters that are nonadaptive with respect to the size of the SE.

In this paper, we discuss and evaluate the suitability of MFs for the derivation of nDSMs from the TDM mission in complex urban environments. More significantly, a novel multistep procedure using progressive morphological image filtering, region growing, and interpolation techniques is described. The approach is designed to yield better results compared to basic MF-based methods in settings where free parameters are nonadaptive with respect to the size of the SE. Moreover, it is intended to lower errors particularly associated with a steep terrain. In this manner, the strategy adopted in this paper aims at a clear reduction of omission errors while taking the risk of a solely moderate increase of commission errors when classifying ground pixels. However, accuracies of classified ground and nonground pixels have a varying influence on the quality of the final $\mathrm{nDSM}$, which is dependent on the terrain characteristics (i.e., flat or steep) of the image domain. To provide a suitable tradeoff between reduction of omission errors and increase of commission errors, regions of pixels that were dominantly classified as nonground by the introduced procedure are spatially refined. Therefore, a postclassification processing scheme is proposed, which adapts techniques of object-based image analysis (OBIA) [30]. To evaluate the suitability of the introduced methods, experiments are carried out with intermediate digital elevation model (IDEM; the characteristics of the data are detailed in Section III-C) data for settlement areas that cover large parts of the cities Izmir (Turkey) and Wuppertal (Germany). We focus on the extraction of elevated objects in urban environments (i.e., buildings), which are characterized by steep terrains and a high relief energy.
The remainder of this paper is organized as follows. Section II details MFs in a consecutive manner: the most basic MF, PMFs, and the proposed region-growing-based PMF (RPMF) procedure are explained in Sections II-A-C, respectively. In Section II-D, the postclassification processing scheme is introduced. Section III is used for the description of data sets and experiments, whereas Section IV reports the actual results of the conducted experiments. Concluding remarks are given in Section V.

\section{MFs Fof DeRIVATION OF NDSMS}

To retrieve heights of objects that are elevated from the Earth's surface such as buildings and vegetation from a DSM, the elevation of the terrain has to be removed. To this purpose, a DTM which represents the BE surface has to be derived first. With this information, an nDSM can be calculated, which contains all objects above the terrain, by subtracting the DTM from the DSM

$$
\mathrm{nDSM}=\mathrm{DSM}-\mathrm{DTM} .
$$

In this way, the DSM is decomposed into terrain (DTM) and object heights (nDSM).

\section{A. Morphological Filter}

A common approach to derive a DTM is to conduct a morphological opening operation on each measurement of the DSM [21], [22]. The approach sequentially executes a minimum and maximum filter with a SE $B_{a}$, e.g., a square window of size $a \times a$, on the grid of the continuous surface elevation measurements $Z$. In the terminology of mathematical morphology, minimum filtering represents an erosion operation extended to grayscale images. It is defined as the minimum of the translations of $Z$ by vectors $-\mathbf{b}$ of $B$ [31, p. 66ff.]

$$
\varepsilon_{B}(Z)=\bigwedge_{\mathbf{b} \in B} Z_{-\mathbf{b}}
$$

Analogously, maximum filtering, which represents a dilation operation, is defined as

$$
\delta_{B}(Z)=\bigvee_{\mathbf{b} \in B} Z_{-\mathbf{b}}
$$

An opening is obtained by the sequential application of a dilation to the result of an erosion

$$
\gamma_{B}(Z)=\delta_{B} \circ \varepsilon_{B}(Z)
$$

To fully eliminate the objects of interest (i.e., buildings) with an opening operation, the size of the SE has to be chosen so that it always exceeds an object's outline. Thus, $B$ must have a side length of $2 \times d_{\max }+1$, with $d_{\max }$ being the half size of the largest object of interest in the number of pixels in the study area, and thus, it can be determined empirically.

Although this classical morphological approach has proven its viability especially in flat terrains [32], it features some conceptual drawbacks. Every single measurement that does 
not represent a minimum in $B$ is altered, and the minimum in $B$ is assigned to it. Therefore, the surface obtained with $\gamma_{B}(Z)$ is usually lower than the original measurements, and object heights are overestimated in the final nDSM [16], [32]. In addition to that, the shape of $B$ (e.g., square or disk) has a considerable influence on the estimation outcome, since it emerges in the resulting image.

\section{B. Progressive Morphological Filter}

To overcome drawbacks related to $\mathrm{MF}$, the PMF method introduces a major methodological difference. It aims to separate $\mathrm{BE}$ pixels from object $(\mathrm{OBJ})$ pixels first and interpolate a DTM from the identified BE pixels. This is done within a progressive procedure, which accounts for different nonground objects with various sizes. To this purpose, an opening operation is conducted on the DSM with an initial size of $B$. Then, a preliminary nDSM (pnDSM; $\Delta Z$ ) is computed with an opening by top-hat operation (OTH)

$$
\mathrm{OTH}=Z-\gamma_{B}(Z)
$$

This allows identifying OBJ pixels by applying an elevation difference threshold $\theta$. In this manner, pixels are classified as OBJ when they are above a certain value for $\theta$. Subsequently, an opening operation can be applied to the DTM with an increased size of $B$ to identify additional OBJ pixels. Therefore, $\theta$ can be varied in dependence of $B$ to address the identification of nonground objects of differing magnitudes (e.g., also in an adaptive way in dependence of the slope of the terrain). The procedure is repeated until a maximum size of $B$ is exceeded. As explained in the previous section, the maximum value for $B$ depends on the size of the largest object of interest. Finally, all unclassified pixels are considered as $\mathrm{BE}$ and can be used for interpolating a DTM [16], [24], [25].

In urban environments, nonground objects primarily consist of bushes, trees, cars, and buildings. Small nonground objects such as cars and trees are removed within the first iterations, while buildings will be removed when they are exceeded by larger sizes of $B$. Analogously, low elevation difference thresholds are used for small sizes of $B$ to eliminate objects such as bushes, small trees, or cars. Larger thresholds are applied for sizes of $B$ which correspond to objects such as buildings. To ensure complete identification of buildings, $\theta$ must be set and maintained to the lowest building height in the study area. As stated in the introductory section, the spatial resolution of the TDM data hampers the exploitation of the conceptual advancement of a progressive procedure. Even the smallest size of $B$ (i.e., $3 \times 3$ pixels) comprises a size that already can easily exceed a building ( $>1200 \mathrm{~m}^{2}$ in our study areas). In such a setting, i.e., using parameters that are nonadaptive with respect to the size of $B$, a progressive procedure cannot be exploited the same way as it can be done for data with ground sampling distances considerably smaller than the objects of interest.

Moreover, a PMFs perform generally well in flat terrains, but at the same time, they are prone to errors in steep terrains, similar to MFs. There are two basic errors that arise in identifying BE pixels in the DSM. One is error of commission (false positives (FPs), type I error) that describes pixels which are mistakenly classified as BE. The second is error of omission that describes pixels that are mistakenly not classified as BE (false negatives (FNs), type II error). In a steep terrain, an increased number of BE pixels are mistakenly identified as OBJ pixels (omission error) because they feature a distinctive difference of the elevation with respect to surrounding pixels similar to, for example, buildings. This leads simultaneously to overestimations of objects and their height in the final nDSM. Generally, the influence of omission and commission errors on the quality of the final nDSM cannot be considered equally and is strongly dependent on the terrain characteristics. For a perfectly flat terrain, a high omission error would yield an acceptable nDSM, since only a few correctly classified BE pixels would allow an adequate interpolation and representation of the terrain. Contrarily, in a steep terrain, the nDSM is prone to contain large fractions of terrain information when BE pixels are not exhaustively identified.

\section{Region-Growing-Based Progressive Morphological Filter}

To address problems particularly associated with nonflat terrain, the strategy adopted here aims at a clear reduction of omission errors while taking the risk of a solely moderate increase of commission errors compared to the PMF approach. An overview of the approach, referred to as RPMF, is given in Fig. 1.

The approach can be subdivided according to three consecutive main steps. First, the DSM is subject to an opening operation with the maximum size of $B$, and $\mathrm{BE}$ pixels are identified by applying an elevation difference threshold. This is done to retain $\mathrm{BE}$ pixels that can be considered reliable (low commission error) but not exhaustive (high omission error). Second, a procedure based on region growing is employed to identify building (i.e., OBJ) pixels from the remaining pixels. Finally, all BE pixels including the identified BE pixels in the first step and non-OBJ pixels in the second step are used for interpolating a DTM, which enables computing the final nDSM.

Regarding the region-growing procedure, initial OBJ pixels are identified first, which are used as seed pixels. Fig. 2 illustrates the image processing steps and criteria applied. The figure is divided according to the strategy adopted for objects smaller (a) and larger (b) than the minimum size of $B$ [the identification of initial OBJ pixels with IDEM TDM data is exemplified in Appendix A, Fig. 8(a)]. To identify objects smaller than $B_{\min }$, a pnDSM is calculated with an opening by top-hat operation. OBJ pixels are identified by the application of a threshold $\theta$ on the pnDSM. An alternative strategy was adopted to account for objects larger than the minimum size of $B$. These objects are fully preserved in the surface obtained with $\gamma_{B_{\min }}(Z)$ and are thus not contained in the pnDSM. Pixels at the border of an elevated object are identified by subtracting the surfaces obtained with $\varepsilon_{B_{\min }}(Z)$ and $\gamma_{B_{\min }}(Z)$. Subsequently, these pixels can be classified as OBJ pixels by sequentially combining an edge extraction filter and contrast segmentation.

In particular, we rely on edge extraction by Lee-sigma edge detection filtering [33]. The Lee-sigma filter represents 


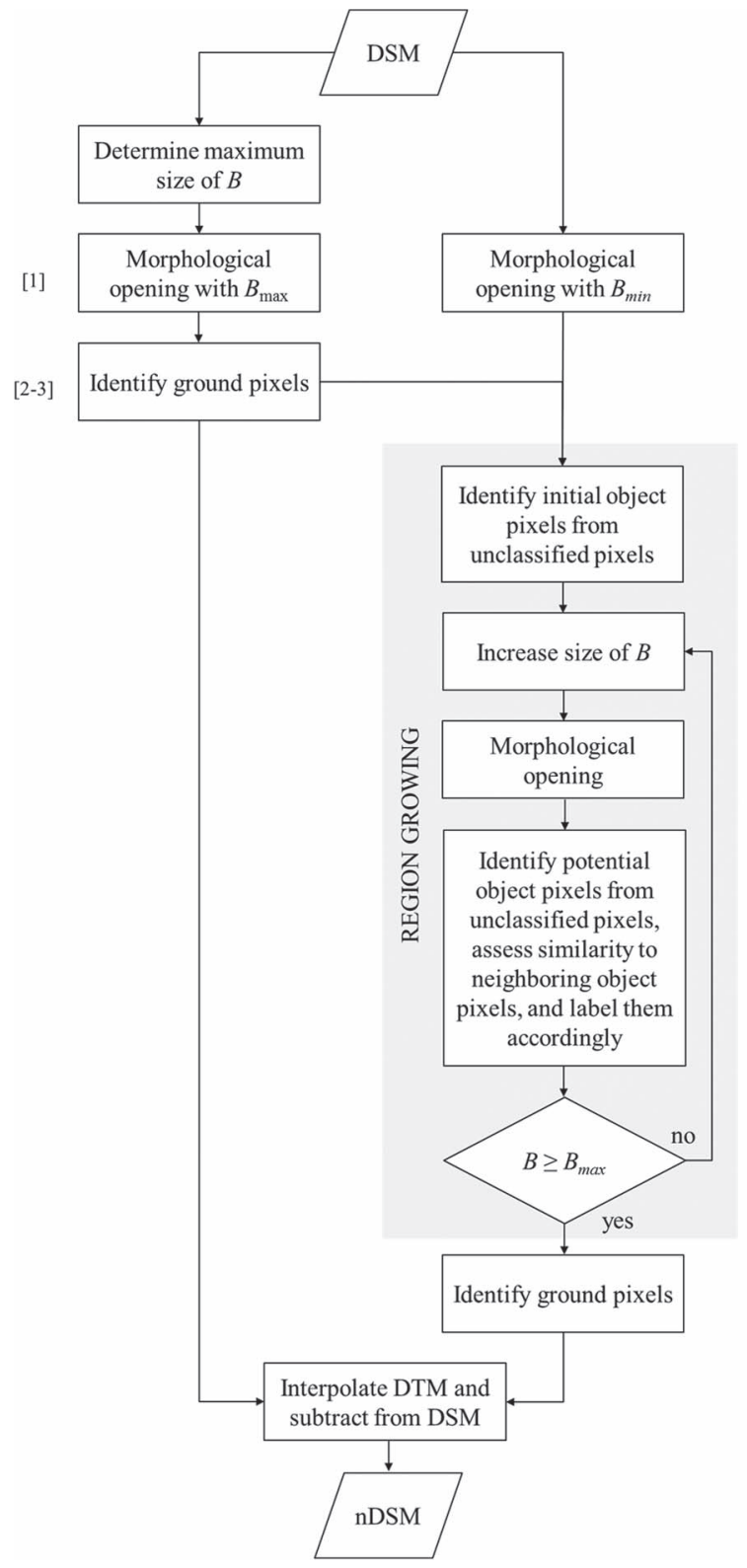

[4]

$[5-8]$

$[9-10,17]$

[11]

$[12-16]$

[18]

[19]

Fig. 1. Flowchart of the RPMF for the derivation of an nDSM from a DSM. Arabic numbers in brackets match the individual steps in the pseudocode of Algorithm 1.

an adaptive filter based upon the spatial domain. It utilizes the sigma probability of the Gaussian distribution to smooth variations in the image by averaging only those neighborhood pixels which have intensities within a fixed range of standard deviations of the center pixel. Consequently, edges and linear features are preserved. The filter is applied on the image data generated by subtracting the surfaces obtained with $\varepsilon_{B_{\min }}(Z)$ and $\gamma_{B_{\min }}(Z)$ to extract bright edges, i.e., distinctive transitions of brightness, which ideally represent boarder pixels of building objects exceeded by $B_{\min }$. To classify these pixels as OBJ in an automated manner, a contrast segmentation approach is deployed, which partitions the filtered image into dark and bright pixels. To maximize the contrast between resulting dark and bright pixels, a threshold is iteratively evaluated and accordingly adjusted. In particular, the threshold is determined by (a) Objects $<B_{\min }$

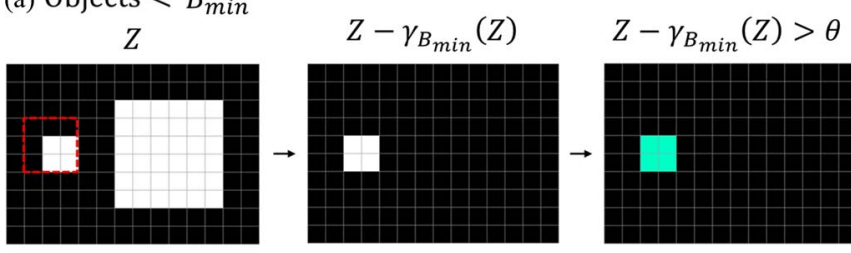

(b) Objects $>B_{\min }$

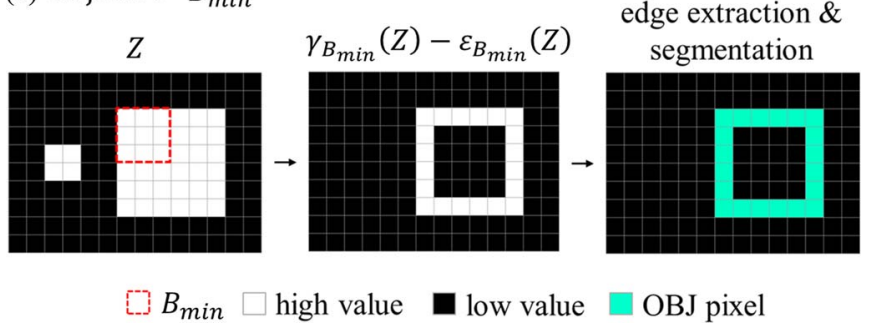

Fig. 2. Procedure for the identification of initial OBJ pixels that represent the basis for the region-growing procedure. (a) Objects that are smaller than the minimum size of $B$ can be identified by the application of a threshold $\theta$ on the pnDSM. (b) Border pixels of objects that are larger than the minimum size of $B$ are identified by subtracting the surfaces obtained with $\varepsilon_{B_{\min }}(Z)$ and $\gamma_{B_{\min }}(Z)$. They are classified as OBJ by combining edge extraction filter and segmentation. Section (a) of this figure visualizes steps (5-6) of Algorithm 1, and section (b) visualizes steps (7-8). The identification of initial OBJ pixels with IDEM TDM data can be seen in Appendix A [Fig. 8(a)].

considering different pixel values as potential thresholds. These potential thresholds range from specified upper and lower bounds, with intermediate values depending on a consecutive step size. Therefore, the contrast is calculated between dark $(q)$ and bright $(r)$ pixels with the term $(r-q) /(r+q)$ [12], [34].

After the identification of initial OBJ pixels that serve as seed pixels, the size of $B$ during the morphological opening is increased linearly

$$
B_{k}=2 \times k+1
$$

where $k=2,3, \ldots, d_{\max }$, with $d_{\max }$ being the largest number of pixels between an object pixel and the next ground pixel (see also Section II-A). In this manner, the filtered surface obtained from the previous iteration is subject to an opening operation with an increased size of $B$. At each iteration, additional OBJ pixels are identified if they exceeded $\theta$ in the respective newly calculated pnDSM and fulfill a similarity constraint with respect to already classified OBJ pixels. The similarity (sim) of a potential OBJ pixel (potOBJ) and adjacent OBJ pixel(s) (OBJ pixels that share a common boundary with a potOBJ pixel analogous to a Queen contiguity [35] or 8-connectivity, respectively) is evaluated as

$$
\operatorname{sim}(\operatorname{potOBJ}, \text { OBJ })= \begin{cases}1, & \left|\mu\left(\Delta Z_{\mathrm{OBJ}}\right)-\Delta Z_{\text {potOBJ }}\right| \leq \eta \\ 0, & \text { else }\end{cases}
$$

where $\Delta Z$ represents the pnDSM value, $\mu$ represents the mean value of neighboring pixels, and $\eta$ represents a threshold. This process is repeated during each iteration until a stable situation is reached, where no more potOBJ pixels are classified as OBJ pixels. Some magnifications of both the identification of initial OBJ pixels that serve as seed pixels as well as of the actual 
region-growing procedure with IDEM TDM data are visualized in Fig. 8(a) and (b), respectively (Appendix A).

The whole procedure to identify BE pixels is also described in the pseudocode under Algorithm 1. Finally, all unclassified pixels are considered as BE and are used in conjunction with previously identified BE pixels to interpolate a final DTM. Therefore, an exact interpolation method, which predicts a value that is equal to the actual value at a sampled location and interpolates solely between values of sampled locations, is favorable to keep elevation values of BE pixels unaltered.

Algorithm 1 Identification of Bare Earth (BE) Pixels With RPMF

Inputs:

$Z$ : A regular grid of continuous surface elevation measurements

$B_{\min }$ : Minimum size of structuring element

$B_{\text {max }}$ : Maximum size of structuring element

$\theta$ : Elevation difference threshold

$\eta$ : Threshold for similarity constraint

Output:

$S$ : Set of pixels that represent ground measurements

1: Conduct $\gamma_{B_{\max }}(Z)=\delta_{B_{\max }} \circ \varepsilon_{B_{\max }}(Z)$

2: Compute $Z-\gamma_{B_{\max }}(Z)$

3: Identify BE pixels that are below $\theta$ and add them to $S$

4: Conduct $\gamma_{B_{\min }}(Z)=\delta_{B_{\min }} \circ \varepsilon_{B_{\min }}(Z)$

5: Compute $Z-\gamma_{B_{\min }}(Z)$

6: Apply $\theta$ to surface obtained with $Z-\gamma_{B_{\min }}(Z)$ and identify initial OBJ pixels that are above $\theta$ and yet unclassified

7: Compute $\gamma_{B_{\min }}(Z)-\varepsilon_{B_{\min }}(Z)$

8: Identify unclassified, initial OBJ pixels by applying edge extraction and contrast segmentation

\section{9: repeat}

10: $\quad$ Initialize $B_{k}$

11: Conduct $\gamma_{B_{k}}(Z)=\delta_{B_{k}} \circ \varepsilon_{B_{k}}(Z)$

12: $\quad$ Compute $Z-\gamma_{B_{k}}(Z)$

13: repeat

14: $\quad$ Apply $\theta$ to surface obtained with $Z-\gamma_{B_{k}}(Z)$ and identify potential OBJ pixels that are above $\theta$, have adjacent OBJ pixel(s), and are yet unclassified

15: Assess similarity of potential OBJ pixels with respect to already identified OBJ pixels with similarity constraint (7) and label them as OBJ pixels when below $\eta$

16: until the number of classified OBJ pixels equals the number of classified OBJ pixels from previous iteration

17: Increment $k$ as described in equation (6)

18: until $B_{k} \leq B_{\max }$

19: Add all unclassified pixels to $S$

\section{Selective Postclassification Processing With OBIA}

As explained in the previous section, the strategy of RPMF is to identify OBJ pixels and subsequently consider all remaining unclassified pixels as BE. This procedure is prone to deliver

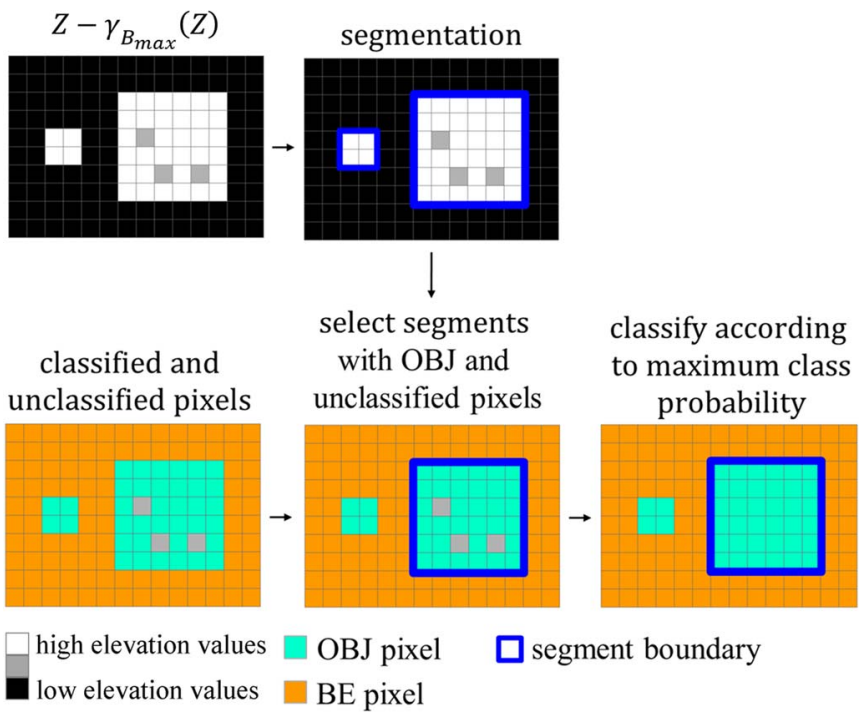

Fig. 3. Scheme for selective postclassification processing of regions with OBJ pixels and unclassified pixels after the region-growing procedure of RPMF. First, a preliminary nDSM is calculated with $B_{\max }$ to ensure that all building objects are contained. A segmentation algorithm is used to discriminate homogenous image regions. Segments are selected, which contain solely OBJ pixels and unclassified pixels. Pixels of selected segments are classified according to the maximum class probability of the respective segment. The selective postclassification processing of IDEM TDM data is exemplified in Appendix B (Fig. 9)

dissatisfactory results when dealing with building objects with irregular roof surfaces or when considerable local variations of elevation values occur, since then the region-growing procedure may not capture all OBJ pixels. Especially when applying small $\eta$ values to the similarity constraint (7) during the regiongrowing procedure, pixels may remain unclassified, although they belong to a continuous building object. Since pixels that remain unclassified after the region-growing procedure will be considered as BE, such errors will result in partially eliminated building objects in the final nDSM. To lower those errors, we introduce a postclassification processing scheme that adapts concepts of OBIA [30], referred to as RPMF with selective object-based voting (RPMF-SOBV). The selective object-based voting scheme is constituted by three consecutive steps illustrated in Fig. 3 and further detailed in the pseudocode of Algorithm 2. 1) The pnDSM computed with $B_{\max }$ is segmented into homogeneous image regions; 2) segments are selected, which contain solely OBJ and unclassified pixels after the region-growing procedure of RPMF; and 3) all pixels of selected segments are classified according to the maximum class probability of the respective segment.

Regarding step 1), the pnDSM is computed with $B_{\max }$ to ensure that all building objects are contained. Hence, segmentation aims at the delineation of building footprints, which are homogeneous in terms of their response in the pnDSM.

We deploy multiresolution segmentation based on the fractal net evolution (FNEA) approach ([36]; implemented in the software environment eCognition). This is a bottom-up regiongrowing segmentation algorithm starting from individual pixels to create image segments of maximum allowable heterogeneity with respect to spectral and geometrical constraints, which need 
to be defined by the user. Therefore, heterogeneity properties are expressed by a scale parameter, which is constituted here by gray-value $\left(h_{\text {color }} \in[0, \ldots, 1]\right)$ and shape heterogeneity $\left(h_{\text {shape }} \in[0, \ldots, 1] ; h_{\text {shape }}=1-h_{\text {color }}\right)$, with shape consisting of smoothness $h_{\text {smooth }}$ and compactness $h_{\text {compact }}$ of the segment boundaries $\left(h_{\text {smooth }}=1-h_{\text {compact }}\right)$. A larger value for the scale parameters induces a less restrictive fusion of adjacent pixels/segments. Hence, modeled segments become larger [37], [38]. Generally, we suggest putting more emphasis on shape heterogeneity rather than on gray-value heterogeneity. This is due to the fact that man-made features such as buildings have distinct shape and size properties, unlike, for example, natural features. Analogously, the weights for heterogeneity of $h_{\text {smooth }}$ and $h_{\text {compact }}$ can be maintained equal. However, the main difficulty lays in the objective determination of an optimal segmentation scale, which can vary in dependence of the size and composition of the objects of an area. We deploy the objective function introduced by the authors of [39] to find domain-specific optimal segmentation scales objectively. Based on the assumption that optimal segmentation maximizes intrasegment homogeneity and intersegment heterogeneity, a measure is calculated by incorporating intrasegment variance $\left(\sigma^{2}\right)$ and Moran's I $(I)$. The intrasegment variance $\left(\sigma^{2}\right)$ with respect to the pnDSM is calculated as

$$
\sigma^{2}=\frac{\sum_{i=1}^{n} A_{i} \sigma^{2}}{\sum_{i=1}^{n} A_{i}}
$$

where $A_{i}$ and $\sigma_{i}^{2}$ represent the area and intrasegment variance of segment $i$. The intrasegment variance $\sigma^{2}$ is the weighted average, with the areas of segments being the weights. As mentioned, Moran's $I$ is used as a measure of intersegment heterogeneity

$$
\begin{aligned}
& I=\frac{N}{\sum_{i} \sum_{j} e_{i j}} \\
& \cdot \frac{\sum_{i} \sum_{j} e_{i j}\left(\mu_{s}(\Delta Z)_{i}-\mu(\Delta Z)\right)\left(\mu_{s}(\Delta Z)_{j}-\mu(\Delta Z)\right)}{\sum_{i}\left(\mu_{s}(\Delta Z)_{i}-\mu(\Delta Z)\right)^{2}}
\end{aligned}
$$

where $N$ is the number of segments indexed by $i$ and $j, \mu_{s}(\Delta \mathrm{Z})$ is the mean pnDSM value of a segment, $\mu(\Delta Z)$ is the mean pnDSM value of all segments, and $e_{i j}$ represents the spatial weight between segments $i$ and $j$ as follows:

$$
e_{i j}= \begin{cases}1, & \text { if } i, j \text { are adjacent neighbor segments } \\ 0, & \text { else. }\end{cases}
$$

With the determined variance and autocorrelation measure, the objective function is calculated by summing up normalized values of $\sigma^{2}$ and $I$

$$
F\left(\sigma^{2}, I\right)=\frac{\sigma_{\max }^{2}-\sigma^{2}}{\sigma_{\max }^{2}-\sigma_{\min }^{2}}+\frac{I_{\max }-I}{I_{\max }-I_{\min }} .
$$

The maximum value of function $F\left(\sigma^{2}, I\right)$ is a statistical indicator of optimal segmentation.
From the generated segments, only those that contain solely OBJ and unclassified pixels after the region-growing procedure of RPMF are selected. For the selected segments, a decision rule is deployed [40], where a probability function is maximized

$$
C(m)=\arg \max _{v \in \mathrm{C}}\left(\tilde{P}_{m, v}\right)
$$

where $C$ is the labeling space constituted by class labels "OBJ" and "unclassified," $C(m)$ represents the final label of pixel $m$, and $\tilde{P}_{m, v}$ denotes the probability of pixel $m$ belonging to class $v$. In accordance with a crisp voting scheme, implemented analogous to a majority vote, we compute

$$
P_{s, v}=\frac{1}{N_{s}} \sum_{u \in \mathrm{s}} \tau(C(u)=v)
$$

where $\tau$ is an indicator function capturing the number of times that the pixels $u$ within a selected segment $s$ feature class label $v$, and $N_{S}$ is the number of pixels in selected segment $s$. The new class label of the pixels in selected segments is determined according to the maximum probability, as indicated in (12).

\footnotetext{
Algorithm 2 Selective Postclassification Processing With RPMF-SOBV
}

\section{Inputs:}

$Z$ : A regular grid of continuous surface elevation measurements

$B_{\max }$ : Maximum window size

$U$ : Set of pixels with class labels "BE", "OBJ", and "unclassified" as determined with the individual steps [1]-[18] in the pseudocode of Algorithm 1

\section{Output:}

$S$ : Set of pixels that represent ground measurements

1: Conduct $\gamma_{B_{\max }}(Z)=\delta_{B_{\max }} \circ \varepsilon_{B_{\max }}(Z)$

2: Compute $Z-\gamma_{B_{\max }}(Z)$

3: Apply segmentation algorithm to preliminary nDSM with different scale factors

4: Compute intrasegment variance and Moran's I with equations (8) and (9), respectively, based on the preliminary $\mathrm{nDSM}$ values of the segments for different segmentation layers

5: Compute objective function with equation (11) to identify optimal segmentation layer

6: Select segments from optimal segmentation layer which contain solely pixels with class labels "OBJ" and "unclassified" in $U$

7: Compute maximum class probability with equations (12) and (13) for selected segments

8: Assign all pixels of selected segments according to respective maximum class probability

9: Add all pixels with class label "unclassified" and "BE" to $S$

The most common processing paradigm of OBIA comprises first a segmentation, followed by an "objectification" (i.e., classification [41]). In contrast to that, RPMF-SOBV relies on per-pixel classification of RPMF, which is followed by segmentation, and then classifies selected image regions based 
on certain class fractions of the superobjects of individual pixels. This procedure is designed to cope naturally with the resolution characteristics of the data, which hampers the use of a pure object-based approach. The latter is frequently intended to model "meaningful image objects" [41]. However, even the smallest spatial entity (i.e., pixel) may correspond to an object of interest (i.e., building). Moreover, in such a setting, a pure object-based approach, comprising the complete aggregation of the image pixels according to certain scale(s), may hardly be applied to TDM data without violating the Shannon sampling theorem. The theorem states that modeled objects should be on the order of one tenth of the dimension of the sampling scheme-the pixel-to ensure that they will be completely independent of their random position and their orientation in relation to the sampling scheme [30]. In this manner, RPMFSOBV is intended to benefit from both the per-pixel and OBIA paradigms. The selective postclassification processing of IDEM TDM data is exemplified in Appendix B (Fig. 9).

\section{DESCRIPTION OF DATA SETS AND Experiments}

\section{A. TanDEM-X IDEM Data}

The TanDEM-X satellite was launched in June 2010, and it is operating jointly in a unique helix tandem formation with its twin radar satellite TerraSAR-X, which is in space since June 2007. Since December 2010, however, the two satellites are operationally acquiring data to generate a seamless global multicoverage digital elevation model using single-pass interferometry in bistatic mode [1]. Since August 2011, the data are being processed operationally. Single- and multibaseline interferometric phase-unwrapping [42], manual quality inspection, water detection [43], height comparison with ICESat data [44], and calibration including tilt and offset correction are carried out in order to provide a homogenous data set for a regional mosaic [45]. In our study, different tiles of the socalled TDM IDEM [46] are used for two test sites. The IDEM consists only of the best quality single-baseline processed data of the first global coverage. Insufficient acquisitions affected by phase-unwrapping errors are excluded. The data sets cover large parts of the settlement areas of the cities Izmir (Turkey; tiles N38E026 and N38E027) and Wuppertal (Germany; tile N51E007). The terrain features high relief energy with partially steep areas in both test sites. The spatial resolution of the IDEM data corresponds to $\sim 12 \mathrm{~m}$ in Izmir and $\sim 11 \mathrm{~m}$ in Wuppertal.

\section{B. UF Data}

To focus our experimental analysis on urban environments, an approach which was introduced by [47] was deployed to discriminate "built-up" and "nonbuilt-up" land cover. The approach was implemented as a fully automated image analysis procedure, which is currently applied to delineate urban footprints (UF) from single polarized strip map imagery of the TDM [48] globally. The high classification accuracy, which exceeds consistently an overall accuracy of $94 \%$ and a $\kappa$ statistic of 0.75 for representative case studies [48], allows us to spatially focus on man-made structures within urban environments (i.e., buildings) and at the same time neglect conceptual and methodological considerations related to other objects that are elevated from the Earth's surface (i.e., vegetation). The deployed UFs of Izmir and Wuppertal cover approximately $214 \mathrm{~km}^{2}$ and $45 \mathrm{~km}^{2}$, respectively, and comprise a large range of different man-made structures, e.g., in Izmir, these reach from small and very low rise informal settlements (Gecekondular) to large industrial buildings.

\section{Description of Experiments}

Experimental analyses and validation are based on two different data sets. 1) The first reference data set contains verified $\mathrm{BE}$ and $\mathrm{OBJ}$ pixels. Here, 1000 points were randomly generated within the $214 \mathrm{~km}^{2}$ of the UF of Izmir. By careful manual inspection of the TDM data and additional utilization of VHR optical imagery, $605 \mathrm{BE}$ pixels and 186 OBJ pixels in the TDM data could be identified. The 209 remaining pixels could not be unambiguously allocated and thus were excluded from the reference data set. 2) The second data set is a DTM derived from LiDAR measurements over the city of Wuppertal. A DTM was derived from the last pulse acquisitions and provided by the city municipality for this study. The experimental analyses are organized in three main parts.

1) The first analysis aims at demonstrating the effectiveness of the proposed RPMF and RPMF-SOBV approaches to separate BE from OBJ pixels for the settlement area of Izmir. Therefore, a comparison with the PMF approach is provided in terms of computed FN and FP rates. The approaches' accuracies are studied as a function of their free parameters.

2) In the second analysis, the accuracies of the generated DTMs are assessed. The qualities are assessed by a comparison with the LiDAR DTM of Wuppertal. Therefore, the deviations of the generated DTMs from the LiDAR DTM were calculated. To this purpose, the LiDAR data and TDM data were coregistered (by means of a nearest neighbor interpolation for resampling) so that the data sets feature a common spatial geometry of $11 \mathrm{~m}$. Moreover, an existing offset of the LiDAR DTM was corrected to match the elevation level of the TDM data by constantly adding $50.4 \mathrm{~m}$ to all measurements. The comparison is based on 370592 pixels, which are identified as settlement area of Wuppertal by the UF data set.

3) In the third analysis, we visually assess the quality of the final $\mathrm{nDSMs}$ according to the different DTM generation approaches and discuss favorable and less favorable characteristics of the respective strategies.

\section{EXPERIMENTAL RESULTS}

\section{A. Results of Analysis I: Accuracy Assessment of BE and OBJ Pixel Classification}

This analysis assesses the ability of PMF, RPMF, and RPMFSOBV to distinguish BE and OBJ pixels. MF is not considered, since it does not feature the inherent concept of the other approaches to separate BE from OBJ pixels first and interpolate a DTM from the identified BE pixels. The classification 
TABLE I

BASIC PARAMETERS FOR PMF, RPMF, AND RPMF-SOBV USED IN THE EXPERIMENTS

\begin{tabular}{c|c|c|c|c|c|c}
\hline Filter approach & $\begin{array}{c}\text { Shape of } \\
\text { structuring } \\
\text { element }\end{array}$ & $\begin{array}{c}B_{\min } \\
{[\text { pixels] }}\end{array}$ & $\begin{array}{c}\text { Increment steps for } \\
\text { the increase of } B \\
(k \text { in Equation }(4))\end{array}$ & $\begin{array}{c}B_{\max } \\
{[\text { pixels] }}\end{array}$ & $\begin{array}{c}\theta \\
\text { [meters] }\end{array}$ & $\begin{array}{c}\eta \\
{[\text { meters] }}\end{array}$ \\
\hline PMF & square & 3 & $2,3, \ldots, 7$ & 15 & $2,2.2, \ldots, 3.6$ & - \\
\hline RPMF & square & 3 & $2,3, \ldots, 7$ & 15 & $2,2.2, \ldots, 3.6$ & $0.6,0.8, \ldots, 2$ \\
\hline RPMF-SOBV & square & 3 & $2,3, \ldots, 7$ & 15 & $2,2.2, \ldots, 3.6$ & $0.6,0.8, \ldots, 2$ \\
\hline
\end{tabular}

accuracies of PMF, RPMF, and RPMF-SOBV were examined by a series of test runs with various values of free parameters $\theta$ and $\eta$. These two free parameters were kept constant during the progressive increase of the size of $B$ for an individual test. This is important since free parameters are frequently altered in dependence of the size of $B$ when working with data with a very high spatial resolution. As explained in Sections I and II-A, low $\theta$ values are used for small sizes of $B$ to eliminate objects such as bushes, small trees, or cars. With increasing size of $B, \theta$ values are also increased to account for objects such as buildings. However, regarding the TDM data, even the smallest size of $B$ $(3 \times 3$ pixels $)$ comprises a size that already can easily exceed a building. To take account for large industrial buildings in the southeastern part of the study area, the maximum size of $B$ was set to 15 pixels for all approaches. Additionally, we chose a square-shaped SE. The height threshold was assessed in the interval $\theta \in[2,2.2, \ldots, 3.6]$, and the similarity constraint was assessed in the interval $\eta \in[0.6,0.8, \ldots, 2]$, whereas a certain combination of $\theta$ and $\eta$ was used and kept constant during each run. The basic parameters of the different approaches are also listed in Table I.

Regarding the edge extraction filter procedure, which is part of RPMF (and then naturally also relevant for RPMF-SOBV), we apply a $\sigma$ value of 4 (preliminary experiments showed that results are insensitive with respect to the specification of $\sigma$ ). Regarding the segmentation which is an essential processing step of RPMF-SOBV, we put more emphasis on shape heterogeneity (shape: 0.7$)$ rather than on gray-value heterogeneity $(0.5)$ as suggested. To identify an optimal segmentation scale that can be used for RPMF-SOBV, we calculated the objective function in terms of equation (11) for scales in the range of $[5, \ldots, 15]$ when applying the segmentation algorithm to the pnDSM computed with $B_{\max }$. The affiliated objective function is revealed in Fig. 4. It can be seen that the maximum value, which represents a statistical indicator for optimal segmentation, is reached with a scale factor of 9 for our data set. Hence, the segmentation used for RPMF-SOBV was carried out with this scale factor.

Based on the reference data, FN and FP rates are calculated for PMF, RPMF, and RPMF-SOBV with the specified parameters. Results are revealed in Fig. 5. In Fig. 5(a), it can be seen that FN rates decrease continuously with an increasing $\theta$ value, and FP rates increase continuously with increasing $\theta$ value for PMF, RPMF, and RPMF-SOBV. This is reasonable since many building objects are lower than a high $\theta$ value in this range, which leads to an increase of FP. Analogously, the terrain is predominantly classified as elevated objects with a low $\theta$ value that results in a high FN rate. Generally, it can be observed that

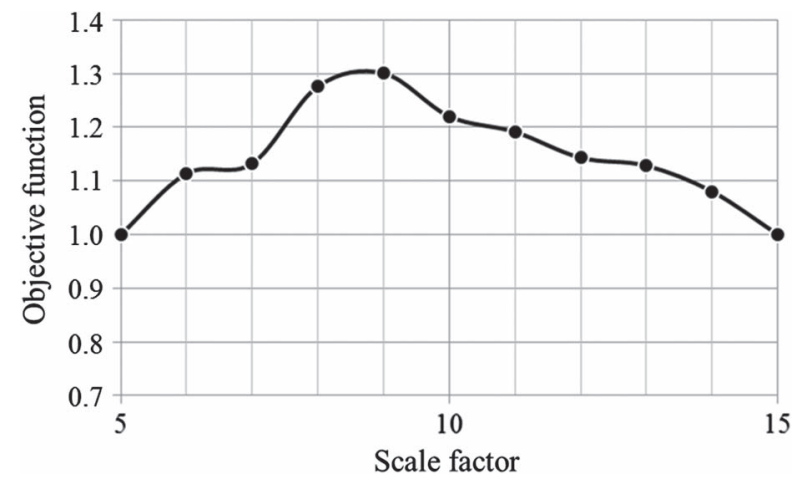

Fig. 4. Objective function calculated according to (8)-(11) for scales in the range of $[5, \ldots, 15]$ when applying the segmentation algorithm to the pnDSM computed with $B_{\max }$. The maximum value of the objective function is a statistical indicator for optimal segmentation. As can be seen, the maximum value for our data set is reached with a scale factor of 9 .

all approaches face the problem of misclassifying the terrain as elevated objects, which results in a high level of FN rates.

When comparing PMF and RPMF, it is obvious that the FN rates of RPMF are consistently lower, and simultaneously, the FP rates are consistently higher. The FN rates of RPMF-SOBV are slightly higher than the FN rates of RPMF, and the FP rates are slightly above the FP rates of PMF. This uncovers an interesting performance characteristic of RPMF-SOBV, which is also exemplified in Fig. 5(b). The figure shows the FN and FP rates for a certain $\theta$ value $(2.6 \mathrm{~m})$ as a function of $\eta$. It can be seen that RPMF-SOBV combines favorable performance characteristics of both PMF and RPMF: regarding FN rates, it is aligned to the better performance of RPMF. In contrast, regarding FP rates, it is aligned to the better performance of PMF. Generally, when increasing $\eta$, we observe that the FN and FP rates associated with RPMF and RPMF-SOBV become more aligned with the error rates of PMF for the given interval. In addition, the sensitivity of the FN and FP rates decreases. Fig. 5(c) visualizes the gain of accuracy (decrease of FN rate) and loss of accuracy (increase of FP rate) of both RPMF and RPMF-SOBV compared to PMF. Generally, it can be observed that the decrease in FN exceeds the increase of FP for both RPMF and RPMF-SOBV compared to PMF explicitly. Compared to RPMF, the decrease of the FN rates of RPMFSOBV is more moderate; however, the increase of FP remains simultaneously on a very low level.

As already mentioned, the classification errors have a varying influence on the quality of the DTM. It is primarily dependent on the terrain characteristics (i.e., flat or steep). Hence, further 
(a)

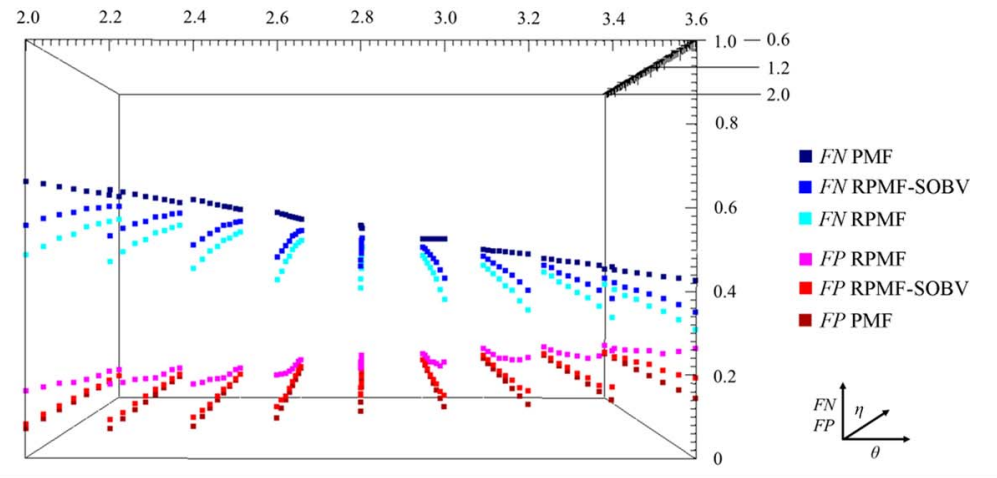

(b)

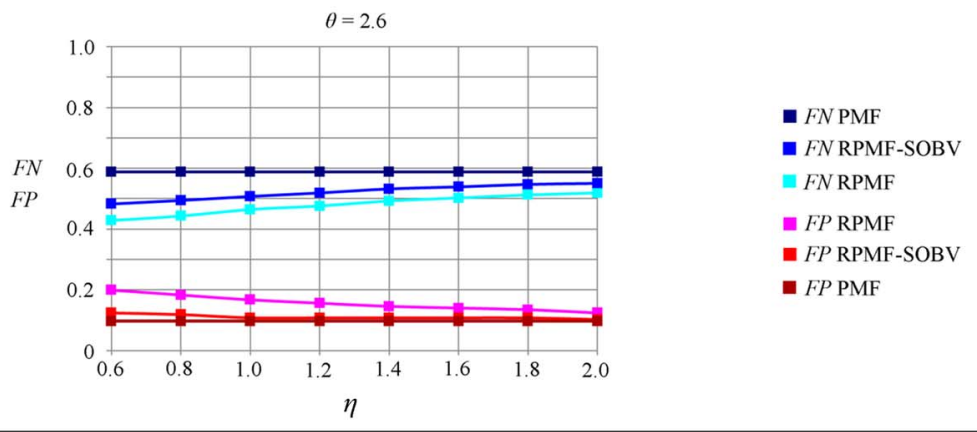

(c)
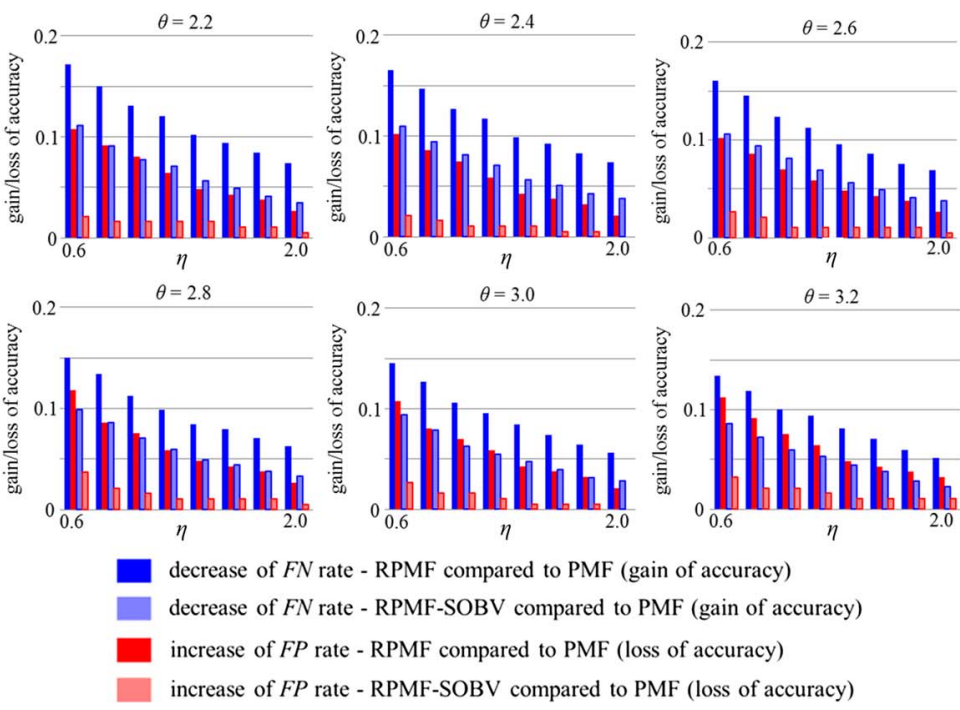

Fig. 5. (a) FN and FP rates achieved with PMF, RPMF, and RPMF-SOBV (plotted on the $y$-axis) in dependence of the approaches' free parameters. The free parameters are height threshold $\theta$ (plotted on the $x$-axis in meters) for PMF, RPMF, and RPMF-SOBV, and similarity constraint $\eta$ (plotted on the $z$-axis in meters; since this parameter is only relevant for RPMF and RPMF-SOBV, it does not vary for PMF). (b) FN and FP rates for a certain $\theta$ value (2.6 m) as a function of $\eta$. (c) Gain of accuracy (decrease of FN rate) and loss of accuracy (increase of FP rate) of both RPMF and RPMF-SOBV compared to PMF.

analysis related to the quality assessment of the resulting DTMs is carried out in the next section.

Moreover, we provide run times needed to classify the BE and OBJ pixels in the DSM for the different algorithms. All algorithms were run under the eCognition Developer environment (version 8.9) on a PC with an Intel Xenon processor at $3.4 \mathrm{GHz}$ and $8-\mathrm{GB}$ RAM. We considered a subset of the IDEM of Izmir consisting of $100 \times 100$ pixels for the comparison. The corresponding run times are as follows: $0.078 \mathrm{~s}$ for PMF, $0.530 \mathrm{~s}$ for RPMF, and $1.217 \mathrm{~s}$ for RPMF-SOBV. Consequently, the observed run times increase in a consecutive manner with the complexity (i.e., processing steps) of the different algorithms. However, depending on the considered problem, the gain in accuracy may justify a higher computational burden.

\section{B. Results of Analysis II: Accuracy Assessment of DTMs}

DTMs were generated based on the BE pixels classified by PMF, RPMF, and RPMF-SOBV. For the Wuppertal data set, we used a maximum size of $B$ of 11 pixels and a scale of 7 for the segmentation algorithm (as determined with the objective function; only relevant for RPMF-SOBV). Additionally, the surface obtained by conducting an opening operation with $B_{\max }(\mathrm{MF})$ is evaluated. A quite restrictive $\theta$ value (i.e., $2.6 \mathrm{~m}$ ) for PMF, RPMF, and RPMF-SOBV was chosen to account for 

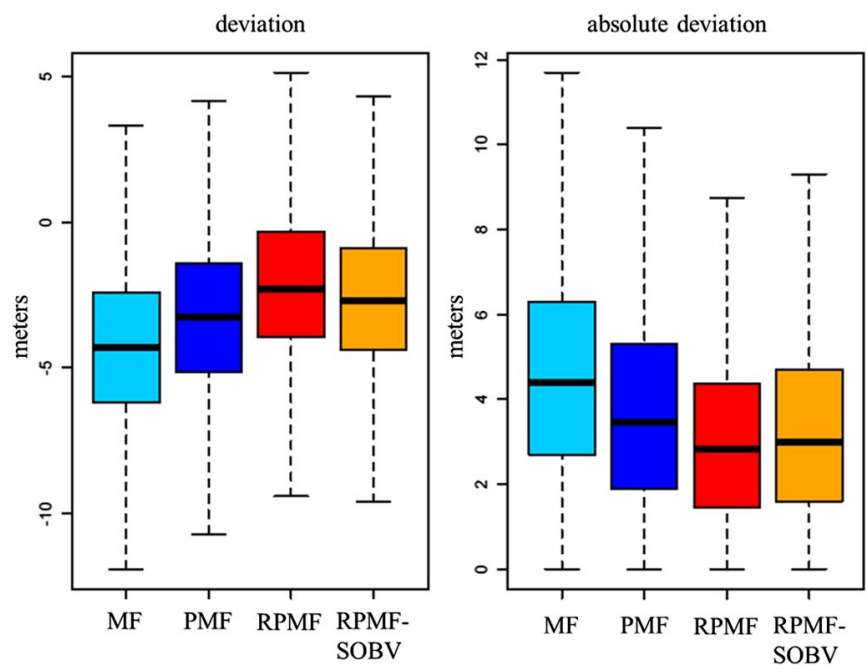

Fig. 6. Boxplots (depicting median, IQR, as well as 1.5 times IQR) for illustration of deviations and absolute deviations of the DTMs with respect to the BE pixels of the TDM data in meters.

the very small and low buildings, as the general aim is to extract every single building. A quite restrictive value for the similarity constraint $\eta$ (i.e., $0.8 \mathrm{~m}$; only relevant for RPMF and RPMFSOBV) was chosen to achieve a favorable tradeoff regarding the overall gain of accuracy with respect to PMF. Analogous to the authors of [16], we used a Kriging approach [49] to interpolate the BE pixels to a DTM surface. We used ordinary Kriging on the BE pixel sets with spherical semivariogram models and adaptive search radii to ensure a number of $12 \mathrm{BE}$ pixels to be included.

As explained in Section III-C, the quality of the generated DTMs is assessed based on the elevation values of 370592 LiDAR DTM pixels. This assessment is intended to serve as a further comparison of methods. Deviations and absolute deviations in meters are visualized in Fig. 6 on the basis of boxplots (depicting median, interquartile range (IQR), as well as 1.5 times IQR). It can be observed that, in general, all approaches have the tendency to underestimate terrain heights compared to the LiDAR measurements. However, RPMF shows consistently for both error measures the smallest deviations with, for example, lowest medians and spreads of IQR. It is followed by RPMF-SOBV, with just slightly higher deviations. The deviations of PMF are higher, and MF shows least favorable performance characteristics with, for example, highest medians and largest spreads of IQR. These results also reflect the results of analysis I. The ability to identify terrain exhaustively is expressed in a low FN rate. In analysis I, RPMF showed the lowest FN rates, followed by RPMF-SOBV and PMF [see Fig. 5(a)].

Moreover, we calculated the mean error (ME), mean absolute error (MAE), root-mean-square error (rmse), and linear deviations at the 90th percentile ( $\mathrm{LD}_{\mathrm{P} 90}$; the results are shown in Table II). In accordance with the results depicted in Fig. 6, RPMF shows the lowest deviations with respect to MAE $(3.27 \mathrm{~m}), \mathrm{ME}(-1.9 \mathrm{~m})$, and rmse $(4.18 \mathrm{~m})$. It is directly followed by RPMF-SOBV, which shows slightly worse quality measures. PMF comes up with a MAE of $3.98 \mathrm{~m}$, a ME of
TABLE II

ME, MAE, RMSE, and Linear Deviations at the 90Th Percentile (LDp90) OF the Different ApProaches in Meters

\begin{tabular}{l|c|c|c|c}
\hline approach & MAE & ME & RMSE & LD 90 \\
\hline MF & 4.75 & -4.24 & 5.58 & 8.4 \\
\hline PMF & 3.98 & -3.29 & 4.97 & 7.59 \\
\hline RPMF & 3.27 & -1.9 & 4.18 & 6.21 \\
\hline RPMF-SOBV & 3.5 & -2.59 & 4.44 & 6.7 \\
\hline
\end{tabular}

$-3.29 \mathrm{~m}$, and an rmse of $4.97 \mathrm{~m}$. These values are above RPMF and RPMF-SOBV but more favorable compared to the values of $\mathrm{MF}$, which shows the highest deviations. $\mathrm{LD}_{\mathrm{P} 90}$ reveals that $90 \%$ of the deviations are smaller than 6.21 and $6.7 \mathrm{~m}$ when using RPMF and RPMF-SOBV, respectively, compared to considerably higher deviations for PMF (7.59 m) and MF (8.4 m). Overall, the results of this analysis are consistent with the results of analysis I and suggest that RPMF features a superior ability to identify terrain with a comparatively low error of omission. Nevertheless, it is directly followed by RPMF-SOBV. A more distinct decrease of performance can be observed when using PMF. MF generally shows least favorable accuracies.

\section{Results of Analysis III: Visual Assessment of nDSMs}

The generated DTMs were subtracted from the TDM DSM to visually assess the quality of the resulting nDSMs. When values smaller than zero occurred in the resulting $\mathrm{nDSM}$, these values were set to zero since they represent artifacts related to the DTM calculation method. "Nonurban" areas are faded out by integrating UF data.

Computed nDSMs are visualized for the Izmir test site in Fig. 7(a) for focus areas in steep (1) as well as flat (2) terrain. Additionally, magnification (3) is intended to exemplify a situation, which motivates the use of RPMF-SOBV. It focuses on large buildings with irregular roof surfaces. The first column shows the VHR optical imagery for comparison. Subsequent to the optical imagery, nDSMs based on MF, PMF, RPMF, and RPMF-SOBV are presented. It can be observed that the overall height levels of the objects differ notably, with MF showing the highest level and RPMF the lowest.

An overestimation of objects' heights by MF and PMF can be dominantly observed in steep terrain (1), also partly for RPMFSOBV. In the central part of the focus area, coherent regions with exaggerated object heights appear. These are caused by $\mathrm{BE}$ pixels that were misclassified as OBJ pixels (the height difference to neighbor pixels is high due to the steepness of the terrain). In such a situation, the interpolation procedure cannot rely on sufficient $\mathrm{BE}$ pixels and thus underestimates the terrain heights. This causation is also revealed in the corresponding height profile (1) in part (b) of the figure (shaded relief with profile lines in yellow is shown in the last section of the upper part). The terrain heights of MF, PMF, and RPMFSOBV purport too low levels, whereas RPMF follows the actual terrain height very closely. In flat terrain [height profile 2 in (b)], a general characteristic of MF becomes obvious as this approach frequently produces terrain models with a too low height level. PMF follows the terrain height more closely and 
(a)
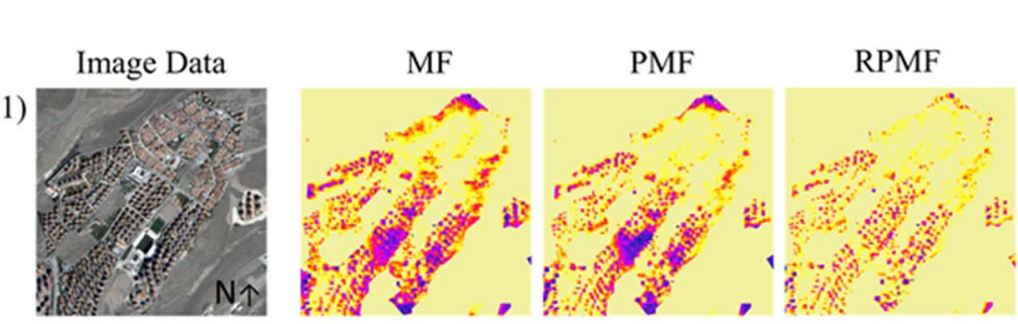

Shaded relief with
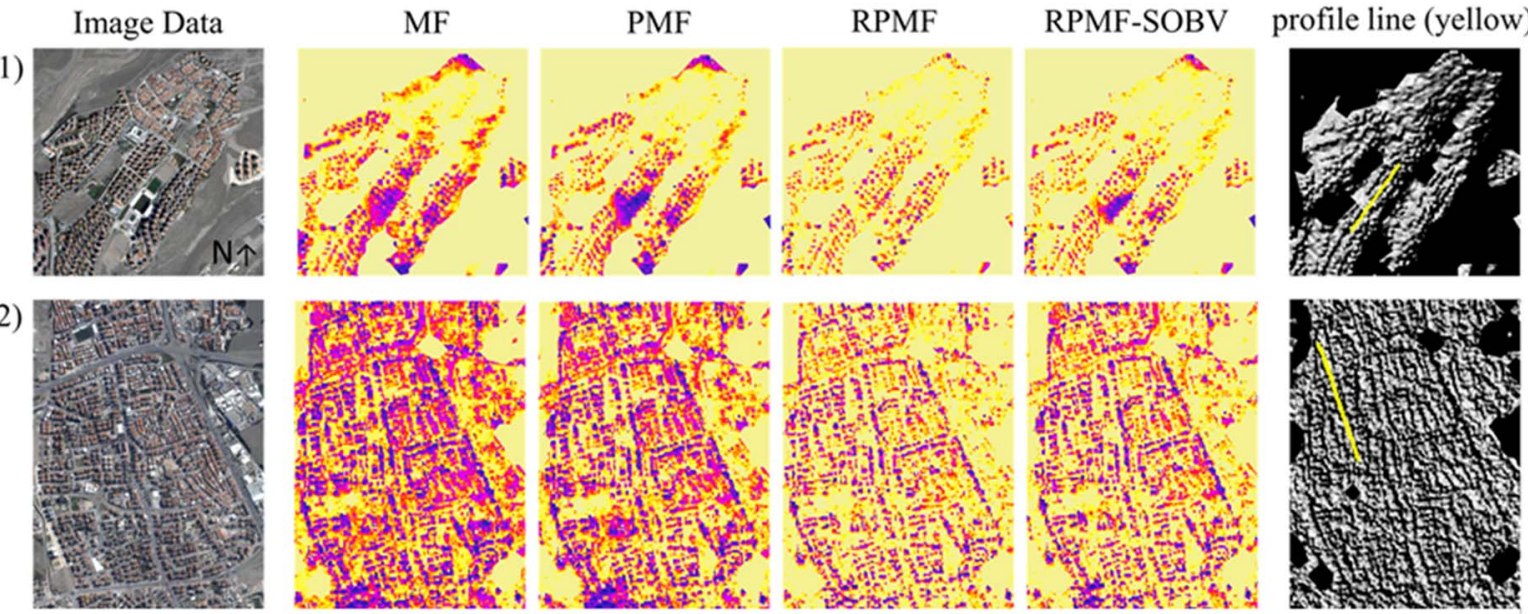

3)
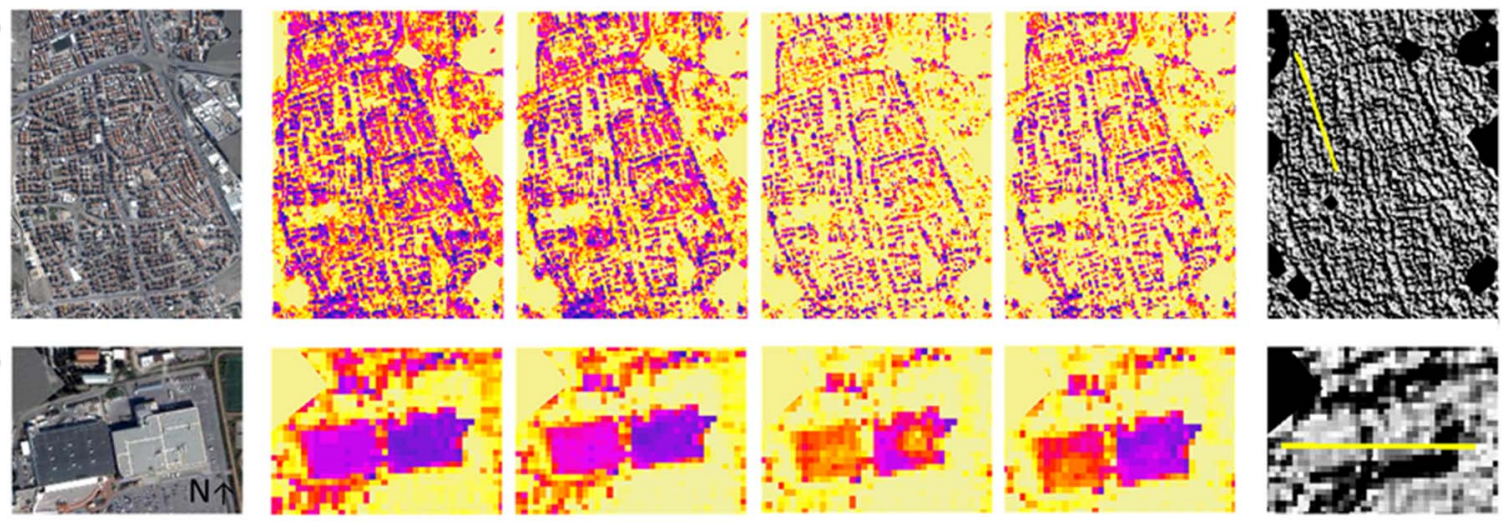

Height [m]

$\leq 0.51$

5

$10 \quad 15 \geq 30$

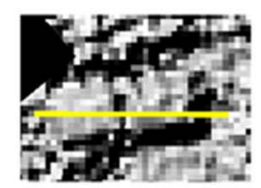

(b)

Profile 1

Height $[\mathrm{m}]$

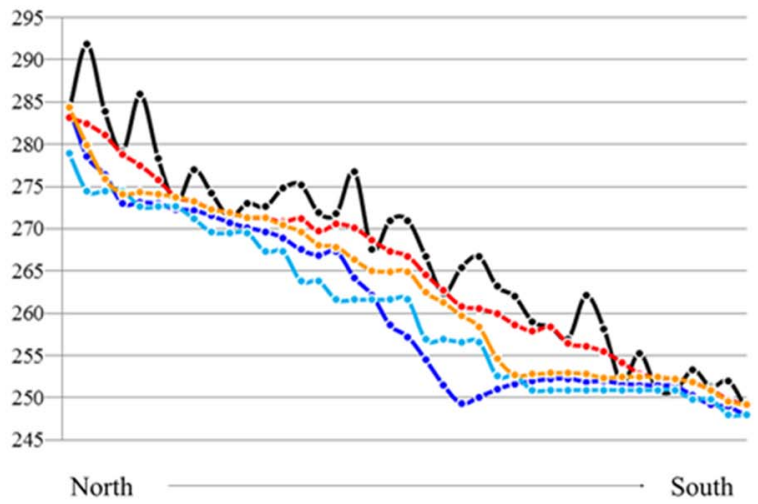

Profile 3

Height $[\mathrm{m}]$

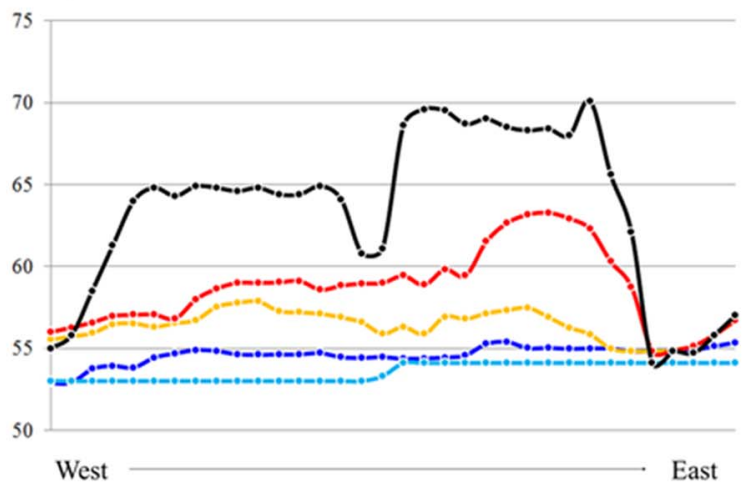

Profile 2

Height $[\mathrm{m}]$

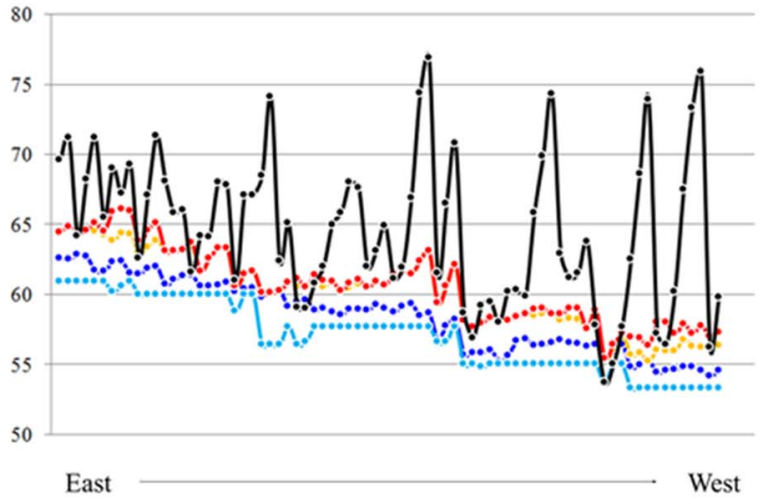

Fig. 7. (a) Visual comparisons of nDSMs based on MF, PMF, RPMF, and RPMF-SOBV with particular focus on (1) steep terrain, (2) flat terrain, and (3) large buildings (which motivated the use of RPMF-SOBV). (b) Affiliated height profiles of DTM surfaces generated by the different approaches. 
(a)

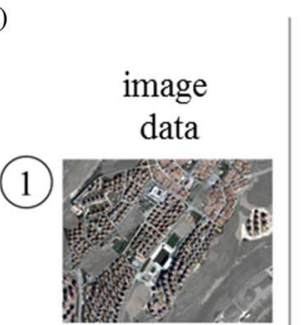

(2)

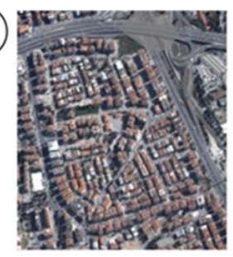

(3)

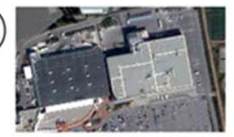

Objects $<B_{\min }$

$Z-\gamma_{B_{3}}(Z) \quad Z-\gamma_{B_{3}}(Z)>\theta \gamma_{B_{3}}(Z)-\varepsilon_{B_{3}}(Z)$
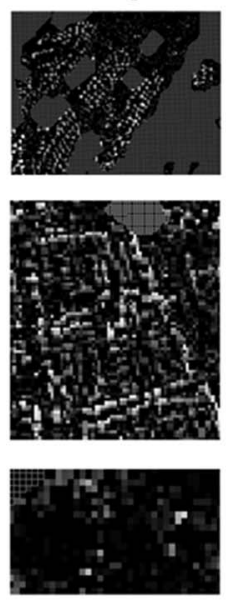
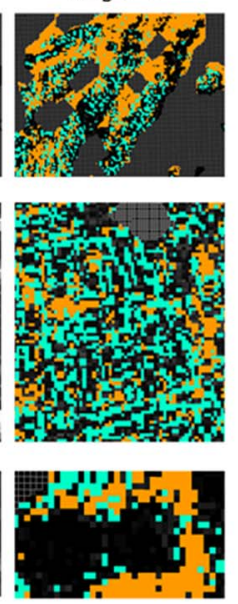
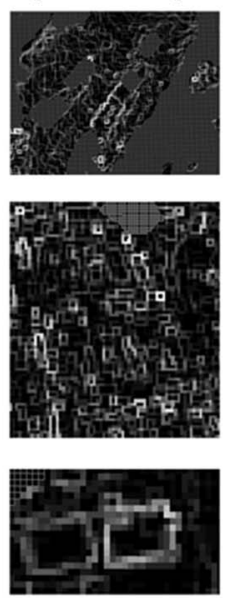

Objects $>B_{\min }$

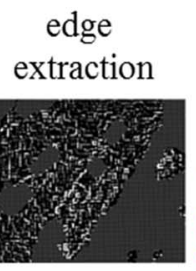

contrast segmentation
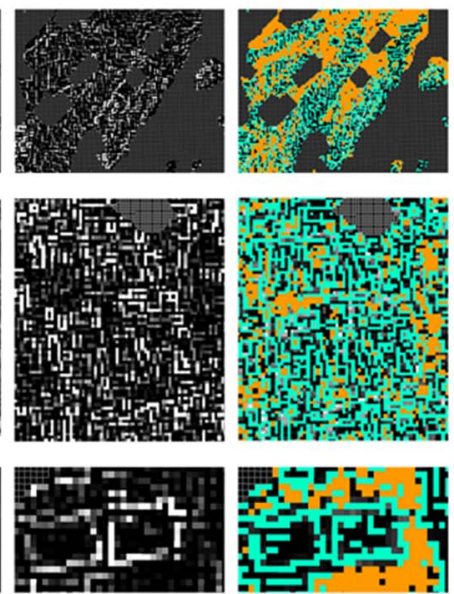

initial OBJ pixels

for region growing
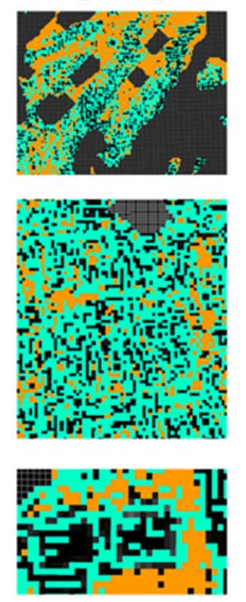

Height $[\mathrm{m}]$

$\leq 0.51$ $\begin{array}{lll}5 & 10 \quad 15 \geq 30\end{array}$

- Non-urban pixel

OBJ pixel

BE pixel

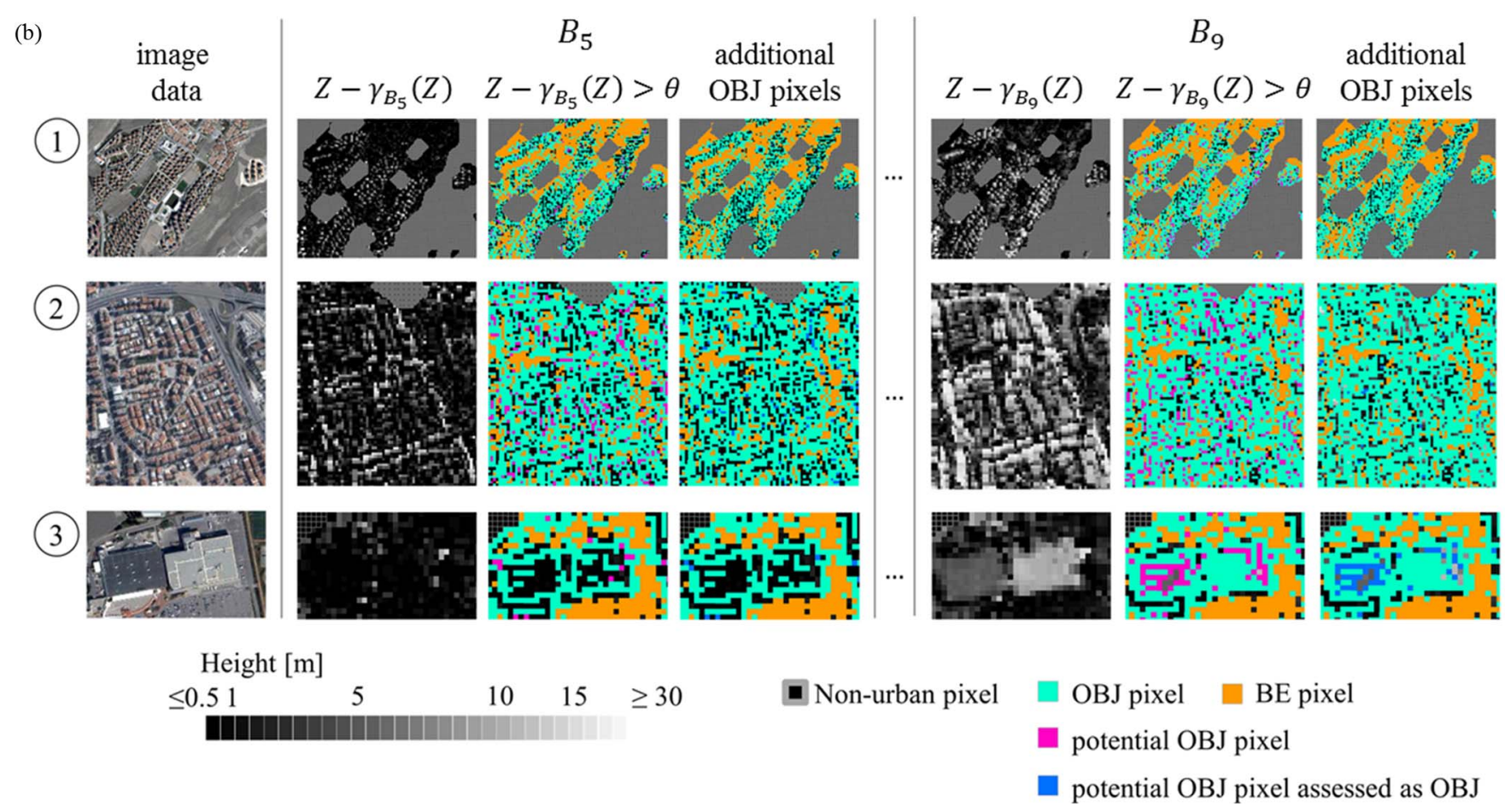

Fig. 8. Exemplification of the procedure for the identification of initial OBJ pixels (a) that represent the basis for the region-growing procedure, which is shown in (b) with IDEM TDM data. In this example, free parameters $\theta$ and $\eta$ were set to 2.6 and $0.8 \mathrm{~m}$, respectively.

naturally. However, the profile reveals a fractional underestimation of the actual terrain heights. On the contrary, RPMF and RPMF-SOBV hardly underestimate terrain heights. The surface generated by RPMF partially reflects a volatile behavior of the height values of the DSM. This can be related to FP errors which cause regions in the interpolated surface that are elevated toward the DSM surface. Interestingly, the surface generated based on RPMF-SOBV appears to be less prone to this behavior. This is specifically illustrated in height profile 3 in (b). It can be observed that the terrain surface produced by RPMF is considerably elevated toward the DSM surface, which leads to underestimated height values in the nDSM as can be seen in section (a), magnification 3. This resulted from the OBJ pixels that remained unclassified during the region-growing procedure and were finally considered as BE. Generally, this error source motivated the introduction of RPMF-SOBV. As 


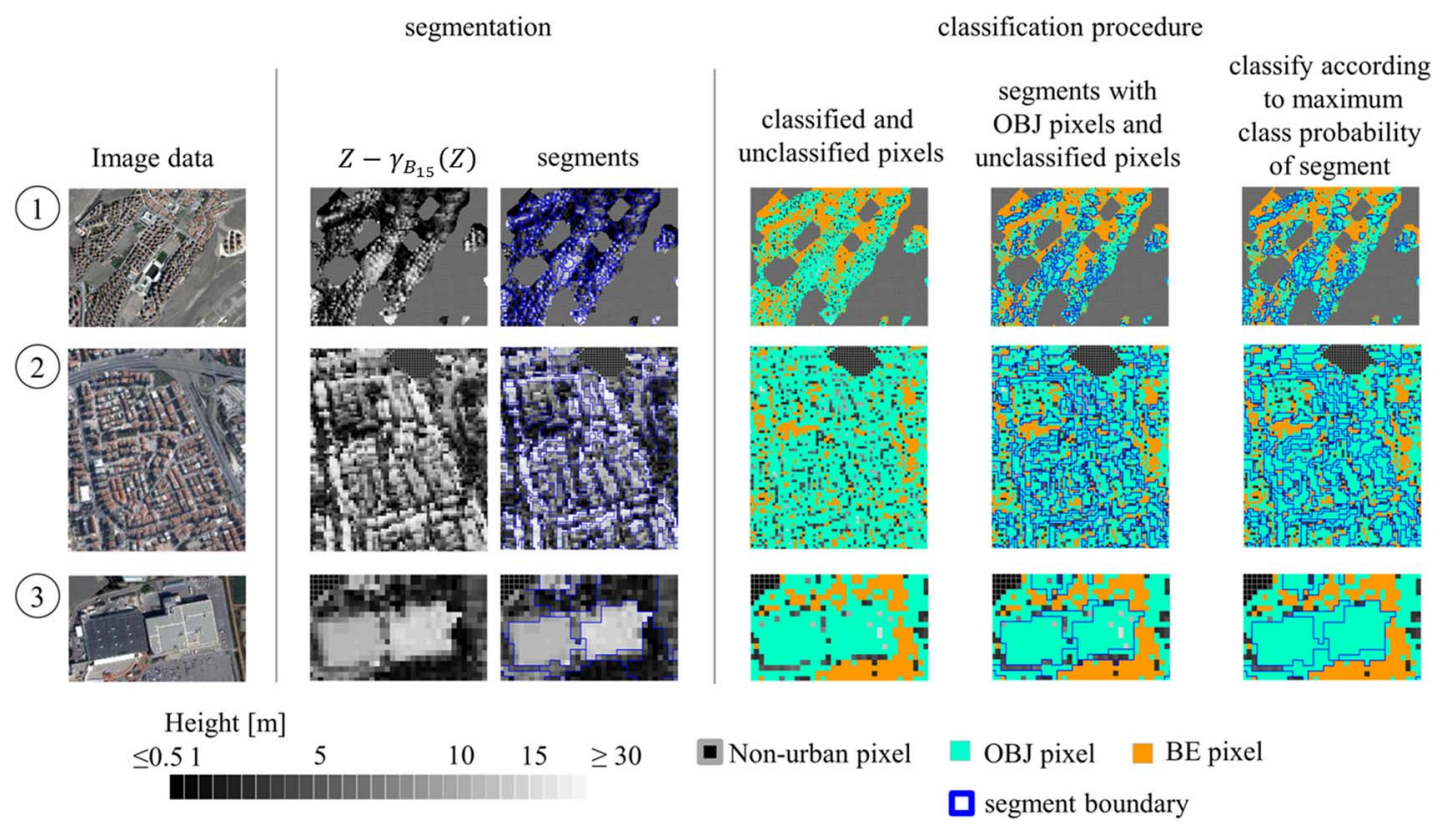

Fig. 9. Exemplified selective postclassification processing scheme. In this example, $B_{\max }$ corresponds to 15 pixels, the preliminary nDSM data were segmented with a scale factor of 9 , and free parameters $\theta$ and $\eta$ were set to 2.6 and $0.8 \mathrm{~m}$, respectively.

can be seen from this example, such errors can be largely avoided with RPMF-SOBV, and more valid surface estimations are achievable.

Overall, results are unambiguously in line with the results of analyses I and II and reflect the capability of the approaches to distinguish $\mathrm{BE}$ from elevated objects correctly. In this manner, the level and proportions of FN and FP rates as evaluated explicitly in analysis I are directly reflected in the computed nDSMs. Hence, analyses suggest that RPMF and RPMF-SOBV yield more favorable performance characteristics compared to PMF and MF in nonflat terrain. In that sense, RPMF features a distinct capability to identify BE pixels in an exhaustive manner, which is advantageous in a steep terrain. Nevertheless, the method may perform less advantageous in situations where the elevation values of the elevated objects feature a volatile behavior. In such situations, RPMF is prone to omit classifying the OBJ pixels. This motivated the introduction of the postclassification processing scheme to spatially refine the classification outcomes of the region-growing procedure of RPMF. Therefore, analyses uncover a notable characteristic of RPMF-SOBV: it yields a favorable tradeoff between a clear decrease of omission errors while keeping errors of commission on a very low level. This makes the approach relevant in various settings.

\section{CONCLUSION}

In this paper, we have proposed a novel RPMF procedure with a postclassification processing scheme for the calculation of nDSMs from TanDEM-X data in urban environments. The proposed methods allow for an accurate reconstruction of urban morphology in challenging terrains. The former is based on a multistep procedure, which sequentially and iteratively executes progressive morphological image filtering and region growing to identify ground pixels. These are subsequently used for the interpolation of a DTM which allows normalizing the DSM. The postclassification processing scheme adapts techniques of OBIA to spatially refine regions of classified nonground pixels.

Experiments were carried out with IDEM data for settlement areas that cover large parts of the cities Izmir (Turkey) and Wuppertal (Germany). Results confirm the interest of the proposed approaches and reveal beneficial performance characteristics compared to basic MF-based approaches. This is evident in a terrain with high relief energy and steep areas. In particular, when classifying ground pixels, RPMF features a clear decrease of omission errors which exceeds an increase of commission errors compared to a basic PMF procedure. The decrease of the omission errors of RPMF-SOBV is more moderate compared to RPMF; however, the increase of the commission errors remains simultaneously on a very low level. This renders the approach relevant for an application in various settings.

The TanDEM-X mission will deliver a globally consistent DSM with an unprecedented spatial resolution. Having suitable methods available for an automated extraction of objects above ground in urban environments will open a broad range of area-wide applications. These are related to the analysis and monitoring of urbanization processes, characterization of urban morphology, and natural disaster mitigation, among others. Hence, valuable contributions to different fields of research may be achieved when extracting relevant information based on presented data and methods.

\section{APPENDIX A}

Fig. 8 shows the exemplifications of the identification of the initial OBJ pixels (a), which are deployed for the regiongrowing procedure (b). Fig. 8(a) illustrates how objects which are smaller than the minimum size of $B$ can be identified by the application of a threshold $\theta$ on the pnDSM. In contrast, 
border pixels of objects that are larger than the minimum size of $B$ are identified by computing $\gamma_{B_{\min }}(Z)-\varepsilon_{B_{\min }}(Z)$. They are classified as OBJ by combining edge extraction filter and segmentation. These initial OBJ pixels are used for the region-growing procedure shown in Fig. 8(b). The size of $B$ is increased, and additional OBJ pixels are identified if they exceeded $\theta$ in the respective newly calculated pnDSM and fulfill a similarity constraint with respect to already classified OBJ pixels.

\section{APPENDIX B}

Fig. 9 shows an exemplification of the selective postclassification processing scheme. First, a preliminary nDSM is calculated with $B_{\max }$ to ensure that all building objects are contained. Subsequently, a segmentation algorithm is used to discriminate homogenous image regions. Segments are selected, which contain solely pixels with class labels "OBJ" and "unclassified" after the region-growing procedure of RPMF. Pixels of selected segments are classified according to the maximum class probability of the respective segment.

\section{ACKNOWLEDGMENT}

The authors would like to thank the city of Wuppertal (Germany) for providing the LiDAR DTM reference data, A. Schmitt and M. Habermeyer (DLR) for the remarks on the initial paper, and the anonymous reviewers for their very helpful comments and suggestions.

\section{REFERENCES}

[1] G. Krieger et al., "TanDEM-X: A satellite formation for high-resolution SAR interferometry," IEEE Trans. Geosci. Remote Sens., vol. 45, no. 11, pp. 3317-3341, Nov. 2007.

[2] P. Griffith, P. Hostert, O. Gruebner, and S. van der Linden, "Mapping megacity growth with multi-sensor data," Remote Sens. Environ., vol. 114, no. 2, pp. 426-439, Feb. 2010.

[3] H. Taubenbock et al., "Monitoring urbanization in mega cities from space," Remote Sens. Environ., vol. 117, pp. 162-176, Feb. 2012.

[4] H. Taubenbock et al., "New dimensions of urban landscapes: The spatiotemporal evolution from a polynuclei area to a mega-region based on remote sensing data," Appl. Geogr., vol. 47, pp. 137-153, Feb. 2014.

[5] I. Baud, M. Kuffer, K. Pfeffer, R. Sliuzas, and S. Karuppannan, "Understanding heterogeneity in metropolitan India: The added value of remote sensing data for analyzing sub-standard residential areas," Int. J. Appl. Earth Observ. Geoinf., vol. 12, no. 5, pp. 359-374, Oct. 2010.

[6] J. Graesser et al., "Image based characterization of formal and informal neighborhoods in an urban landscape," IEEE J. Sel. Topics Appl. Earth Observ. Remote Sens., vol. 5, no. 4, pp. 1164-1176, Aug. 2012.

[7] H. Taubenbock et al., "Delineation of central business districts in mega city regions using remotely sensed data," Remote Sens. Environ., vol. 136, pp. 386-401, Sep. 2013.

[8] C. Geiß and H. Taubenbock, "Remote sensing contributing to assess earthquake risk: From a literature review towards a roadmap," Nat. Hazards, vol. 68, no. 1, pp. 7-48, Aug. 2013.

[9] M. Pittore and M. Wieland, "Towards a rapid probabilistic seismic vulnerability assessment using satellite and ground-based remote sensing," Nat. Hazards, vol. 68, no. 1, pp. 115-145, Aug. 2013.

[10] C. Geiß et al., "Assessment of seismic building vulnerability from space," Earthquake Spectra, vol. 30, no. 4, pp. 1553-1584, Nov. 2014.

[11] B. Sirmacek, H. Taubenbock, B. Reinartz, and M. Ehlers, "Performance evaluation for 3-D city model generation of six different DSMs from airand spaceborne sensors," IEEE J. Sel. Topics Appl. Earth Observ., vol. 5, no. 1, pp. 59-70, Feb. 2012.

[12] M. Wurm, H. Taubenbock, M. Schardt, T. Esch, and S. Dech, "Objectbased image information fusion using multisensor Earth observation data over urban areas," Int. J. Image Data Fusion, vol. 2, no. 2, pp. 121-147, Jun. 2011
[13] P. Gamba and B. Houshmand, "Digital surface models and building extraction: A comparison of IFSAR and LiDAR data," IEEE Trans. Geosci. Remote Sens., vol. 38, no. 4, pp. 1959-1968, Jul. 2000.

[14] K. Kraus and N. Pfeiffer, "Determination of terrain models in wooded areas with airborne laser scanner data," ISPRS J. Photogramm., vol. 53, no. 4, pp. 193-203, Aug. 1998.

[15] P. Axelsson, "Processing of laser scanner data-Algorithms and applications," ISPRS J. Photogramm., vol. 54, 2/3, pp. 138-147, Jul. 1999.

[16] K. Zhang et al., "A progressive morphological filter for removing nonground measurements from airborne LiDAR data," IEEE Trans. Geosci. Remote Sens., vol. 41, no. 4, pp. 872-882, Apr. 2003.

[17] G. Sithole and G. Vosselman, "Experimental comparison of filter algorithms for bare-Earth extraction from airborne laser scanning point clouds," ISPRS J. Photogramm., vol. 59, 1/2, pp. 85-101, Aug. 2004.

[18] X. Liu, "Airborne LiDAR for DEM generation: Some critical issues," Progr. Phys. Geogr., vol. 32, no. 1, pp. 31-49, Feb. 2008.

[19] G. Vosselman, "Slope based filtering of laser altimetry data," in Proc. Int. Arch. Photogramm. Remote Sens., 2000, vol. B4/33, pp. 958-964.

[20] G. Sithole, "Filtering of laser altimetry data using a slope adaptive filter," in Proc. Int. Arch. Photogramm. Remote Sens. Spatial Inf. Sci., 2001, vol. XXXIV (3/W4), pp. 203-210.

[21] R. Haralick, S. Sternberg, and X. Zhuang "Image analysis using mathematical morphology," IEEE Trans. Pattern Anal. Mach. Intell., vol. PAMI-9, no. 4, pp. 532-550, Jul. 1987.

[22] U. Weidner and W. Forstner, "Towards automatic building extraction from high resolution digital elevation models," ISPRS J. Photogramm., vol. 50, no. 4, pp. 38-49, Aug. 1995.

[23] J. Kilian, N. Haala, and M. Englich, "Capture and elevation of airborne laser scanner data," in Proc. Int. Arch. Photogramm. Remote Sens. Spatial Inf. Sci., 1996, vol. 31, Pt. B3, pp. 383-388.

[24] Q. Chen, P. Gong, D. Baldocchi, and G. Xie, "Filtering airborne laser scanning data with morphological methods," Photogramm. Eng. Remote Sens., vol. 73, no. 2, pp. 175-185, Feb. 2007.

[25] T. J. Pingel, K. C. Clarke, and W. A. McBride, "An improved simple morphological filter for the terrain classification of airborne LiDAR data," ISPRS J. Photogramm., vol. 77, pp. 21-30, Mar. 2013.

[26] R. M. Haralick and L. G. Shapiro, Computer and Robot Vision. Reading, MA, USA: Addison-Wesley, 1991.

[27] X. Meng, N. Currit, and K. Zhao, "Ground filtering algorithms for airborne LiDAR data: A review of critical issues," Remote Sens., vol. 2, no. 3, pp. 833-860, Mar. 2010.

[28] A. S. Maguya, V. Junttila, and T. Kauranne, "Adaptive algorithm for large scale DTM interpolation from LiDAR data for forestry applications in steep forested terrain," ISPRS J. Photogramm., vol. 85, pp. 74-83, Nov. 2013.

[29] S. Mayer, "Extraction of tree groups from high-resolution digital surface models," in Proc. Int. Conf. Image Process., Vancouver, BC, Canada, Sep. 10-13, 2000, vol. 3, pp. 712-715.

[30] T. Blaschke, "Object based image analysis for remote sensing," ISPRS J. Photogramm., vol. 65, no. 1, pp. 2-16, Jan. 2010.

[31] P. Soille, Morphological Image Analysis, 2nd ed. Berlin, Germany: Springer-Verlag, 2004.

[32] T. Krauss, H. Arefi, and P. Reinartz, "Evaluation of selected methods for extracting digital terrain models from satellite borne digital surface models in urban areas," in Proc. Int. Conf. SMPR, 2011, pp. 1-7.

[33] J.-S. Lee, "Digital image smoothing and the sigma filter," Comput. Vis. Graph., vol. 24, no. 2, pp. 255-269, Nov. 1983.

[34] Trimble eCognition Developer 8.8 Reference Book, Trimble Germany $\mathrm{GmbH}$, Munich, Germany, 2012.

[35] M. Stasolla and P. Gamba, "Spatial indexes for the extraction of formal and informal human settlements from high-resolution SAR images," IEEE J. Sel. Topics Appl. Earth Observ., vol. 1, no. 2, pp. 98-106, Jun. 2008.

[36] U. C. Benz, P. Hofmann, G. Willhauck, I. Lingenfelder, and M. Heynen, "Multi-resolution, object-oriented fuzzy analysis of remote sensing data for GIS-ready information," ISPRS J. Photogramm. Remote Sens., vol. 58, no. 3/4, pp. 239-258, Jan. 2004.

[37] S. Martinis, A. Twele, and S. Voigt, "Unsupervised extraction of floodinduced backscatter changes in SAR data using Markov image modeling on irregular graphs," IEEE Trans. Geosci. Remote Sens., vol. 49, no. 1, pp. 251-263, Jan. 2011.

[38] T. Esch, M. Thiel, M. Bock, A. Roth, and S. Dech, "Improvement of image segmentation accuracy based on multiscale optimization procedure," IEEE Geosci. Remote Sens. Lett., vol. 5, no. 3, pp. 463-467, Jul. 2008.

[39] G. M. Espindola, G. Camara, I. A. Reis, L. S. Bins, and A. M. Monteiro, "Parameter selection for region-growing image segmentation algorithms using spatial autocorrelation," Int. J. Remote Sens., vol. 27, no. 14, pp. 3035-3040, Jul. 2006. 
[40] X. Huang, Q. Lu, L. Zhang, and A. Plaza, "New postprocessing methods for remote sensing image classification: A systematic study," IEEE Trans. Geosci. Remote Sens., vol. 52, no. 11, pp. 7140-7159, Nov. 2014.

[41] T. Blaschke et al., "Geographic object-based image analysis-Towards a new paradigm," ISPRS J. Photogramm. Remote Sens., vol. 87, pp. 180-191, Jan. 2014.

[42] C. Rossi, F. R. Gonzalez, T. Fritz, N. Yague-Martinez, and M. Eineder, "TanDEM-X calibrated raw DEM generation," ISPRS J. Photogramm., vol. 73, pp. 12-20, Sep. 2012.

[43] A. Wendleder et al., "TanDEM-X water indication mask: Generation and first evaluation results," IEEE J. Sel. Topics Appl. Earth Observ. Remote Sens., vol. 6, no. 1, pp. 171-179, Feb. 2012.

[44] M. Huber et al., "Ensuring globally the TanDEM-X height accuracy: Analysis of the reference data sets ICESat, SRTM and KGPS-tracks," in Proc. IEEE IGARSS, Cape Town, South Africa, 2009, pp. 769-772.

[45] A. Gruber, B. Wessel, M. Huber, and A. Roth, "Operational TanDEM-X DEM calibration and first validation results," ISPRS J. Photogramm., vol. 73, pp. 39-49, Sep. 2012

[46] B. Wessel et al., "TanDEM-X Ground Segment DEM Products Specification document" Earth Observation Center, Weßling, Germany, TD-GS-PS-0021, no. 2.0, Apr. 22, 2013. [Online]. Available: https://tandemx-science.dlr.de/

[47] T. Esch et al., "Delineation of urban footprints from TerraSAR-X data by analyzing speckle characteristics and intensity information," IEEE Trans. Geosci. Remote Sens., vol. 48, no. 2, pp. 905-916, Feb. 2010.

[48] T. Esch et al., "Urban footprint processor-Fully automated processing chain generating settlement masks from global data of the TanDEM-X mission," IEEE Geosci. Remote Sens. Lett., vol. 10, no. 6, pp. 1617-1621, Nov. 2013.

[49] H. Wackernagel, Multivariate Geostatistics-An Introduction With Applications, 3rd ed., Berlin, Germany: Springer-Verlag, 2003.

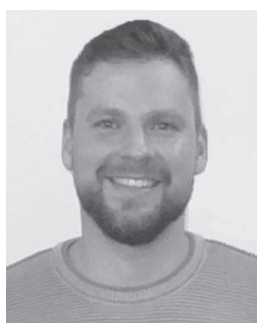

Christian Geiß received the M.Sc. degree in applied geoinformatics from the Paris Lodron University of Salzburg, Salzburg, Austria, in 2010 and the Ph.D. degree (Dr.rer.nat.) from the Humboldt University of Berlin, Berlin, Germany, in 2014.

Since 2010, he has been with the German Remote Sensing Data Center (DFD), German Aerospace Center (DLR), Weßling, Germany. He worked on several topics related to renewable energy, earthquake risk assessment, and methods for remote sensing data processing. His current research interests include techniques of multimodal remote sensing, machine learning, and pattern recognition, specifically applied to the field of natural hazard risk research.

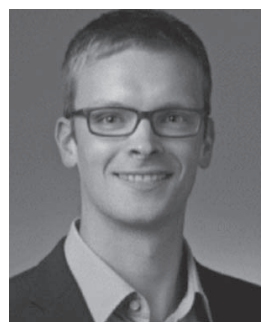

Markus Breunig received the Diploma degree in geography (M.Sc.) from the University of Würzburg, Würzburg, Germany, in 2008.

Since 2009, he has been a Research Associate with the German Remote Sensing Data Center (DFD), German Aerospace Center (DLR), Weßling, Germany. After several topics on urban EO based both on optical and SAR data, his work now focuses on the DEM generation within the TanDEM-X mission. He is operating the DEM production chain, i.e., system monitoring and quality assessment of DEM preparation, calibration, and mosaicking.

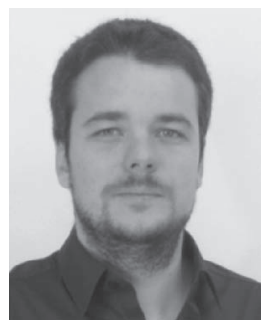

Andreas Felbier received the Dipl.Ing. degree in geodesy and geoinformation from the Technical University of Munich, Munich, Germany, in 2009.

$\mathrm{He}$ is involved in the TanDEM-X project since his diploma thesis, where he developed a methodology to improve the SRTM elevation model by the determination of the quality criteria for the reference data set ICESat. Since 2009, he has been working on the Global Urban Footprint Processor. The main goal of this project is to derive a global map of human settlements based on the data acquired in the context of the TerraSAR-X and TanDEM-X missions. He has experiences in digital image processing and software development.

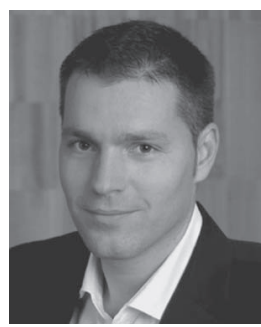

Hannes Taubenböck received the Diploma degree in geography from the Ludwig-Maximilians Universität München, Munich, Germany, in 2004 and the Ph.D. degree (Dr.rer.nat.) in geography from the Julius-Maximilian's University of Würzburg, Würzburg, Germany, in 2008.

In 2005, he joined the German Remote Sensing Data Center (DFD), German Aerospace Center (DLR), Munich, Germany, working on the topic "Mega cities and Natural Disasters." He is a Lecturer with the University of Würzburg, Würzburg. In 2010 , he was a Scientific Employee with DLR-DFD and became the head of the "Modelling and geostatistical methods" team in 2013. His research activities as Postdoctoral Researcher with the University of Würzburg (2007-2010) concentrated on the development of algorithms for information extraction from multisensoral remotely sensed data and subsequent value adding for transforming scientific results into practical value.

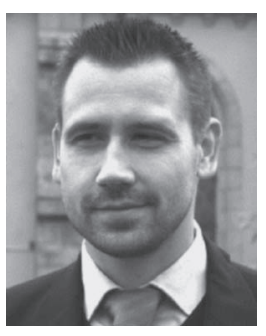

Michael Wurm received the Diploma degree (Mag.rer.nat.) in geography with a specialization on remote sensing, GIS, and spatial research from the University of Graz, Graz, Austria, in 2007 and the $\mathrm{Ph} . \mathrm{D}$. degree (Dr.rer.nat.) in surveying and geoinformation from Graz University of Technology, Graz, in 2013.

$\mathrm{He}$ was with the Institute of Digital Image Processing, Joanneum Research, Graz, in 2007. In 2008, he joined the Remote Sensing Unit, Geography Department, University of Würzburg, Würzburg, Germany, working on the integration of Earth observation data into social sciences and urban remote sensing as well as urban vulnerability research. In 2011, he joined the German Remote Sensing Data Center (DFD), German Aerospace Center (DLR), Weßling, Germany. Working in the research unit "Modelling and geostatistical methods," he focuses on information extraction from multisensoral remote sensing data and its fusion with ancillary data. Until today, he has been contributing to several research projects in urban remote sensing. 